\title{
Dynamics of the spin-half Heisenberg chain at intermediate temperatures
}

\author{
O. A. Starykh, 1 , 畞 A. W. Sandvik ${ }^{2}$, and R. R. P. Singh ${ }^{1}$ \\ ${ }^{1}$ Department of Physics, University of California, Davis, California 95616 \\ ${ }^{2}$ National High Magnetic Field Laboratory, Florida State University, 1800 East Paul Dirac Drive, Tallahasse, FL 32306
}

(June 3, 2021)

\begin{abstract}
Combining high-temperature expansions with the recursion method and quantum Monte Carlo simulations with the maximum entropy method, we study the dynamics of the spin- $1 / 2$ Heisenberg chain at temperatures above and below the coupling $J$. By comparing the two sets of calculations, their relative strengths are assessed. At high temperatures, we find that there is a low-frequency peak in the momentum integrated dynamic structure factor, due to diffusive long-wavelength modes. This peak is rapidly suppressed as the temperature is lowered below $J$. Calculation of the complete dynamic structure factor $S(k, \omega)$ shows how the spectral features associated with the two-spinon continuum develop at low temperatures. We extract the nuclear spin-lattice relaxation rate $1 / T_{1}$ from the $\omega \rightarrow 0$ limit, and compare with recent experimental results for $\mathrm{Sr}_{2} \mathrm{CuO}_{3}$ and $\mathrm{CuGeO}_{3}$. We also discuss the scaling behavior of the dynamic susceptibility, and of the static structure factor $S(k)$ and the static susceptibility $\chi(k)$. We confirm the asymptotic low-temperature forms $S(\pi) \sim$ $[\ln (T)]^{3 / 2}$ and $\chi(\pi) \sim T^{-1}[\ln (T)]^{1 / 2}$, expected from previous theoretical studies.

PACS: 75.10.Jm, 75.40.Gb, 75.50.Ee, 76.60.-k
\end{abstract}

\section{INTRODUCTION}

Quantum antiferromagnets represent an important class of systems in both theoretical and experimental condensed matter physics. In recent years, greatly improved precision of neutron scattering and NMR experiments have made possible very detailed comparisons with theoretical predictions. A number of new materials have been synthesized which appear to benear-perfect realizations of the simple spin-half Heisenberg model in various geometries. For example, $\mathrm{Sr}_{2} \mathrm{CuO}_{3}$, , 2 2 $\mathrm{SrCu}_{2} \mathrm{O}_{3}$, 3 and $\mathrm{Sr}_{2} \mathrm{CuO}_{2} \mathrm{Cl}_{2}, \mathrm{l}$ comprise structural copper-oxygen units with magnetic properties well described by the Heisenberg model on a single 1D chain, two coupled chains, and a 2D square lattice, respectively. $\mathrm{Sr}_{2} \mathrm{CuO}_{3}$ is interesting because it appears to be the most perfect 1D spin-half Heisenberg system found so far, rwith an exchange $J \approx 2000 \mathrm{~K}$ and a $3 \mathrm{D}$ ordering temperature $T_{\mathrm{N}} \approx 5 K$. Detailed experimental studies of this system 1 . 2 as well as other quasi-1D materials such as $\mathrm{KCuF}_{3}$ and $\mathrm{CuGeO}_{3}, \frac{1}{6}$ have pointed to the need for more accurate theoretical studies of the spin dynamics of the $S=1 / 2$ Heisenberg chain. Although this model, defined in standard notation by the Hamiltonian

$$
H=J \sum_{i} \mathbf{S}_{i} \cdot \mathbf{S}_{i+1}, \quad(J>0),
$$

is perhaps the most studied of the basic interacting quantum many-body models, its finite-temperature dynamic properties are not fully understood. The low-temperature $(T \ll J)$ behavior is controlled by the $T=0$ quantum critical point (line of critical points to be exact). The powerful machinery of bosonization and conformal field theory enables one to make a number of experimentally verifiable predictions in this regime. $\mathrm{d}$. The high temperature regime $(T \gg J$ has been studied numerically by short-time or frequency moment expansions combined with the recursion method. 9 The regime of intermediate temperatures $T \approx J$ is the most difficult to study theoretically, but is clearly of much experimental and theoretical significance, containing the crossover from the diffusive high-temperature behavior to the low-temperature regime dominated by elementary-excitations. Here we study the dynamics at intermediate temperatures using the high-temperature expansion (HTE) technique and a recently developed "stochastic series expansion" quantum Monte Carlo (QMC) technique 10 .11 (an improved variant of the so called Handscomb's method 12 ). We have also numerically diagonalized the Hamiltonian for a chain with 16 spins, which although not large enough to give reliable results in general, provides for a good test of the other methods in certain-regimes.

The HTE method has been extensively used to study static properties of spin-models. 3 Here we combine it with the continued fraction (or recursion) method 1 to calculate dynamic properties at finite temperatures. The QMC method used here has also previously been applied to both statics and dynamics of Heisenberg models in several different geometries 14.45 Accurate results for imaginary-time dependent correlation functions can be obtained down to fairly low temperatures. The maximum-entropy (Max-Ent) method 16127 is used for analytic continuation to real frequencies. This approach has previously been applied to the spin dynamics of the 1D Heisenberg model by Deisz, 
Jarrell and Cox 1819 who focused mainly on the low-temperature dynamic structure factor and the differences between half-integer and integer spin. They also discussed at length the accuracy of the Max-Ent method. Here we find that for static properties, results obtained using HTE and QMC agree almost perfectly for $T / J \gtrsim 1 / 8$, below which the HTE method becomes unreliable. For dynamic properties, the HTE method performs well for $T / J \gtrsim 0.5$, and in this regime the results agree well with those of QMC and the Max-Ent method. This gives us confidence that the QMC and Max-Ent techniques are reliable at lower temperatures as well.

Our main results are the following: At high temperatures, we find that the dynamic structure factor $S(k, \omega)$ grows sharply (perhaps diverges) as $k \rightarrow 0$ and $\omega \rightarrow 0$. This diffusive behavior leads to a low-frequency peak in the momentum average

$$
S_{A}(\omega)=\int \frac{d k}{2 \pi}|A(k)|^{2} S(k, \omega)
$$

if the form factor $A(k=0)$ is non-zero. In an NMR experiment, depending on the $A(k)$ corresponding to a giren material and nucleus under study, this can have large effects on the spin-lattice relaxation rate, which is given byed

$$
\frac{1}{T_{1}}=2 S_{A}(\omega \rightarrow 0) .
$$

QMC+Max-Ent results for $1 / T_{1}$ were previously reported in Ref. 15. Here we provide results of higher accuracy, obtained by calculating the full momentum dependence of $S(k, \omega \rightarrow 0)$.

As the temperature is lowered below $T \approx J / 2$, the diffusive peak rapidly diminishes in magnitude, and the lowfrequency spectral weight shifts to $k=\pi$, as expected. The $\mathrm{OAfC}$ results for $S(k, \omega)$ clearly show the emergence of spectral features that can be associated with the well known21 6 . $T=0$ two-spinon continuum.

Comparisons of the momentum and frequency-dependent numerical data with scaling theories at low temperatures have been presented elsewhere.22 Here we briefly discuss how the scaling in $q / T$ is violated due to logarithmic corrections. We provide numerical results for the temperature dependence of the staggered structure factor $S(\pi)$ and the static staggered susceptibility $\chi(\pi)$. At low temperatures the former behaves as $[\ln (1 / T)]^{3 / 2}$, while the latter behaves as $T^{-1}[\ln (1 / T)]^{1 / 2}$, both expected from theoretical results.

In Sec. II we discuss the dynamic structure factor and the computational methods used in this study. The results are presented in Sec. III, and in Sec. IV we discuss and summarize our main conclusions.

\section{BASIC DEFINITIONS AND NUMERICAL TECHNIQUES}

We begin by reviewing some basic definitions of the static and dynamic correlation functions we wish to calculate. Both the neutron scattering intensity and the NMR spin-lattice relaxation rate measure the dynamics of the electronic spin system through coupling via the operator $S_{k}^{+}$. Hence, the relevant dynamic correlation function is $\left\langle S_{-k}^{-}(t) S_{k}^{+}(0)\right\rangle$. In a spin-rotationally invariant system, which is considered here, this can be evaluated with respect to any quantization axis, and in numerical calculations it is most practical to choose the component diagonal in the representation chosen. Hence, we study the time-dependent spin-spin correlation function

$$
S_{r}(t)=\left\langle S_{r}^{z}(t) S_{0}^{z}(0)\right\rangle,
$$

were $S_{r}^{z}(t)$ denotes the $z$-component of a spin-1/2 operator at site $r$ at time $t$, and brackets denote thermodynamic averaging at temperature $T / J=\beta^{-1}$. We consider only the case of zero average magnetization; $\left\langle S_{r}^{z}\right\rangle=0$. The dynamic structure factor $S(k, \omega)$ is the space-time Fourier transform of Eq. (何):

$$
S(k, \omega)=\sum_{r} \int_{-\infty}^{\infty} d t \mathrm{e}^{-i(\omega t-k r)}\left\langle S_{r}^{z}(t) S_{0}^{z}(0)\right\rangle .
$$

Apart from kinematic factors, the neutron scattering cross section is directly proportional to $S(k, \omega)$.

NMR (and related techniques such as NQR) can provide accurate results for the low-frequency dynamics, through the spin-lattice relaxation rate, given by Eqs. (2) and (3). The hyperfine form-factor $A(k)$ can be obtained from the Knight shift, and also from impurity effects.26.27 Here we will restrict our attention to the important case where the nucleus under consideration resides on the sites of the electronic spins, and assume that the real-space hyperfine coupling $\bar{A}(r)$ has a on-site (direct) term $\bar{A}(0)$ and a nearest neighbor (transferred) term $\bar{A}(1)$, giving $A(k)=\bar{A}(0)+$ $2 \bar{A}(1) \cos (k)$. The spin-lattice relaxation rate is then given by 


$$
\frac{1}{T_{1}}=2 \bar{A}^{2}(0) S_{R}\left(\omega=\omega_{N}\right)
$$

where, $R=\bar{A}(1) / \bar{A}(0), \omega_{N}$ is the resonance frequency, and we define

$$
S_{R}(\omega)=\left(1+2 R^{2}\right) S_{0}(\omega)+4 R S_{1}(\omega)+2 R^{2} S_{2}(\omega),
$$

where $S_{r}(\omega)$ is the real space dynamic spin correlation function at distance $r$, i.e., the time Fourier transform of Eq. (1).

The static structure factor $S(k)$ is the Fourier transform of the equal-time correlation function, and the static susceptibility $\chi(k)$ is given by the Kubo integral

$$
\chi(k)=\sum_{r} \mathrm{e}^{i k r} \int_{0}^{\beta} d \tau\left\langle S_{r}^{z}(\tau) S_{0}^{z}(0)\right\rangle,
$$

where $S_{r}^{z}(\tau)=\mathrm{e}^{\tau \hat{H}} S_{r}^{z} \mathrm{e}^{-\tau \hat{H}} . S(k)$ and $\chi(k)$ can be related to the dynamic structure factor through the sum rules 28

$$
\begin{aligned}
& S(k)=\frac{1}{\pi} \int_{0}^{\infty} d \omega\left(1+\mathrm{e}^{-\beta \omega}\right) S(k, \omega), \\
& \chi(k)=\frac{2}{\pi} \int_{0}^{\infty} d \omega \omega^{-1}\left(1-\mathrm{e}^{-\beta \omega}\right) S(k, \omega) .
\end{aligned}
$$

Below we briefly describe the numerical techniques we use to calculate the dynamic structure factor of the 1D Heisenberg model.

\section{A. High Temperature Expansion and the Recursion Method}

The correlation function (4) has a short-time expansion

$$
S_{r}(t)=\sum_{n=0}^{\infty} M_{n} \frac{(-i t)^{n}}{n !}
$$

where the coefficients $M_{n}$ are defined as frequency moments,

$$
M_{n}=\int_{-\infty}^{\infty} \frac{d \omega}{2 \pi} \omega^{n} S_{r}(\omega),
$$

of the time Fourier transform

$$
S_{r}(\omega)=\int_{-\infty}^{\infty} d t \mathrm{e}^{-i \omega t} S_{r}(t)
$$

An important related function is the spectral density $\Phi(\omega)=\left(1+e^{-\beta \omega}\right) S(\omega)$, which is a real and even function of frequency and, consequently, its inverse Fourier transform $C_{0}(t)$ (often called the fluctuation function) has an expansion in even powers of time only:

$$
C_{0}(t)=\sum_{k=0}^{\infty}(-1)^{k} M_{2 k} \frac{(-i t)^{2 k}}{(2 k) !} .
$$

A short-time expansion is of little help if one is interested in the asymptotic long-time behavior of $C_{0}(t)$, unless some kind of analytic ansatz (most often a gaussian one) is made. To this end, let us define the relaxation function $\mathrm{g}$

$$
c_{0}(z)=\int_{0}^{\infty} d t e^{-z t} C_{0}(t)=\sum_{k=0}^{\infty} M_{2 k} z^{-(2 k+1)} .
$$


From Eq. (13), one then has

$$
c_{0}(z)=\int_{-\infty}^{\infty} \frac{d \omega}{2 \pi} \frac{\Phi(\omega)}{z+i \omega}
$$

and $\Phi\left(\omega^{\prime}\right)=2 \lim _{\epsilon \rightarrow+0} \operatorname{Re}\left[c_{0}\left(\epsilon-i \omega^{\prime}\right)\right]$. Thus, upon analytic continuation the relaxation function gives the spectral density. A useful property of the relaxation function is that it has a continued fraction representation:

$$
c_{0}(z)=\frac{\Delta_{0}}{z+\frac{\Delta_{1}}{z+\frac{\Delta_{2}}{z+\ldots}}} .
$$

To simplify notation we shall in the following write the continued fraction as

$$
c_{0}(z)=\Delta_{0} /\left(z+\Delta_{1} /\left(z+\Delta_{2} /(z+\ldots)\right)\right) .
$$

The first $K$ of the continued fraction coefficients are uniquely determined by the corresponding first $K$ even frequency moments (11) through an iterative procedure described in Ref. 9. Of course, we just traded the short-time behavior of $C_{0}(t)$ for the large- $z$ behavior of $c_{0}(z)$, which does not bring us any closer to the desired $z \sim 0$ region. But, as described in detail in the book by Viswanath and Müller 1 the relaxation function is uniquely determined by the sequence $\Delta_{k}$, which contains important information about the asymptotic behavior of $c_{0}(z)$. Namely, for the isotropic Heisenberg model (11), the $\Delta$-sequence grows with the index $k$ according to a power law, $\Delta_{k} \sim k^{\lambda}$, with $1 \leq \lambda \leq 2$, which fixes the high-frequency behavior of the spectral density to $\Phi(\omega) \sim \exp \left(-|\omega|^{2 / \lambda}\right)$. Moreover, oscillations of the odd (or even) continued fraction coefficients around the $k^{\lambda}$ curve contain information on the infrared behavior of $\Phi(\omega)$. The simplest function that incorporates both the high- and low-frequency behavior typical of critical spin systems has the form

$$
\bar{\Phi}(\omega)=\frac{2 \pi}{\lambda \omega_{0} \Gamma(\lambda(1+\alpha) / 2)}\left|\frac{\omega}{\omega_{0}}\right|^{\alpha} \exp \left(-\left|\frac{\omega}{\omega_{0}}\right|^{2 / \lambda}\right) .
$$

The frequency moments of this function are known to be

$$
\bar{M}_{2 k}=\omega_{0}^{2 k} \frac{\Gamma\left(\frac{\lambda}{2}(1+\alpha+2 k)\right)}{\Gamma\left(\frac{\lambda}{2}(1+\alpha)\right)}
$$

and the corresponding continued fraction coefficients $\bar{\Delta}_{k}$ can be calculated from them numerically. Of course, an approximation of the spectral density $\Phi(\omega)$ of the system under study by the model density $\bar{\Phi}(\omega)$, with parameters $\omega_{0}, \alpha, \lambda$ determined from a "given" sequence $\Delta_{k}$, would be just marginally better than the often used gaussian ansatz. Instead, Müller and collaborators (see Ref. 9 and references therein) devised a more accurate procedure, which we describe here for completeness.

Suppose that we have calculated the first $K$ even moments of the true spectral density $\Phi(\omega)$. Then we calculate the corresponding sequence $\Delta_{k}$, and try to approximate it by the model sequence $\bar{\Delta}_{k}$ by minimizing the sum $\sum_{k=k_{m i n}}^{K}\left(\Delta_{k}-\right.$ $\left.\bar{\Delta}_{k}\right)^{2}$ with respect to the parameters $\omega_{0}, \alpha, \lambda$. The lower cut-off $k_{\min }$ ( $=3$ in our study) is necessary because the first few coefficients $\Delta_{0}, \Delta_{1}, \ldots, \Delta_{k_{\text {min }}}$ tend to deviate significantly from the asymptotic behavior represented by $\bar{\Phi}(\omega)$. Having determined the parameters of the fit we may find exactly the $K$-th level terminator $\bar{\Gamma}_{K}(z)$ of the model relaxation function corresponding to $\bar{\Phi}(\omega)$ by (numerically) inverting the equation

$$
\bar{c}_{0}(z)=\bar{\Delta}_{0} /\left(z+\bar{\Delta}_{1} /\left(z+\ldots+\bar{\Delta}_{K} /\left(z+\bar{\Gamma}_{K}(z)\right)\right)\right) .
$$

The terminator thus incorporates information on the asymptotic behavior of the $\bar{\Delta}_{k}$-sequence.

The relaxation function $c_{0}(z)$ in Eq. (17) is then approximated as

$$
c_{0}(z)=\Delta_{0} /\left(z+\Delta_{1} /\left(z+\ldots \Delta_{K} /\left(z+\bar{\Gamma}_{K}(z)\right)\right)\right),
$$

and thus, in addition to the correct large- $z$ behavior contained in the first few exactly known continued fraction coefficients, through the terminator $\bar{\Gamma}_{K}(z), c_{0}(z)$ also incorporates the correct small- $z$ behavior extracted from the $\Delta_{k}$ sequence. Analytic continuation $z \rightarrow-i \omega^{\prime}$ then gives us the spectral function $\Phi\left(\omega^{\prime}\right)$ in the whole range of frequencies $\omega^{\prime}$. For the model spectral function $\bar{\Phi}(\omega)$ of type (18), such analytic continuation is performed by hand and only requires numerical integration of well-behaved functions. 
To study the spin dynamics at finite temperature we have calculated moments of $S_{r}(\omega)$ by the HTE technique. It is well knewn that these moments, $M_{k}$, can be expressed in terms of a thermal expectation value $q$ f a $k$-fold commutator.23. High temperature expansion can be developed for these quantities by the cluster method.24 In fact, using the same set of clusters, the expansions for the $k$-th moment will be complete to order $\beta^{N-k}$, where $N$ depends on the size of the largest cluster considered. We have calculated up to $N=22$ for all non-zero moments. The equal-time correlation function is calculated to order $\beta^{20}$. It is more convenient to do the calculations for the scaled function $c_{0}(z) / M_{0}$, i.e. the one defined by the normalized set of moments $\left\{1, M_{2} / M_{0}, \ldots, M_{2 K} / M_{0}\right\}$, and obtain the needed function $c_{0}(z)$ by simple multiplication at the very end of calculations.

The behavior of the first 7 continued fraction coefficients of the spin autocorrelation function $\left\langle S_{0}^{z}(t)^{z} S_{f}(0)\right\rangle$ as a function of inverse temperature is shown in Fig. 1. From the relation between $M_{2 k}$ and the $\Delta_{k}$ sequenced we have $\Delta_{0}=M_{0}$, and hence $\Delta_{0}=1 / 4$ irrespective of temperature in that case. Another property of the $\Delta_{k}$ 's that make them convenient for numerical computations is that they are all numbers of order 1, whereas the corresponding frequency moments grow very fast with the index, for example $M_{14}(T=\infty)=166988876$. Continued fraction coefficients with higher index $k$ are related to the moments $M_{2 k}$ which are determined by spin correlations at larger distances, and hence are more temperature sensitive. The HTE ceases to work for $\Delta_{7}$ at $\beta \sim 0.75$ where it starts to change rapidly, and for $\Delta_{6}$ at $\beta \sim 1.5$. Since we want to reach the lowest possible temperature, we restrict ourselves to first 6 $(k=0 \ldots 5)$ coefficients of the sequence, which permits analysis up to $\beta \sim 2$. Of course, the shorter the $\Delta_{k}$ sequence the more uncertain becomes the determination of parameters $\omega_{0}, \alpha, \lambda$, and in each particular study a try-and-see approach should be used to find a compromise between these two conflicting requirements. We found that at $\beta \leq 0.5$ results obtained with $K=7$ and $K=5$ do not differ much, and upto $\beta=2$ the sequence $\Delta_{0} \ldots \Delta_{5}$ is stable and reliable.

In Fig. 1 we also show the $k$-dependence of the $\Delta_{k}$ sequence for $S_{R}(\omega)$ (Eq.(7)) for $R=0$ and $R=-0.5$. The latter has a vanishing form factor at $k=0$ and thus has no contributions from the diffusive modes. It is evident that the former sequence exhibits an odd-even oscillation, suggesting an infrared singularity, but this is absent from the latter sequence. This ability to recognize the presence or absence of diffusive modes at such a simple level shows the power of the recursion method.

The temperature dependence of the parameters of the fit, Eq. 18), is shown in Fig. 2. Notice the drastic difference in $\alpha$, the power of the infrared singularity for $R=0$ and $R=-0.5$ : in the latter case it is always zero, whereas in the former it decreases rapidly with temperature, demonstrating the suppression of the diffusive behavior at low $T$.

The overall quality of the described procedure can be estimated by direct term-to-term comparison of the real sequence $\Delta_{k}$ with the model one $\bar{\Delta}_{k}$, given in Fig. 3 for $k=3,4,5$. Deviations of $\bar{\Delta}_{k}$ from $\Delta_{k}$ are most pronounced at high temperatures for the $R=0$ case, where the low-frequency infrared divergence is the strongest, and almost disappear for $T$ below $J$. The deviations at high $T$ can be reduced by working with longer $\Delta_{k}$-sequences, as was mentioned above, but this would significantly reduce the range of applicability of our calculations. The absolute value of the discrepancy between $\Delta$ 's does not exceed $5 \%$ in the worst case.

\section{B. Stochastic Series Expansions and the Maximum Entropy Method}

The stochastic series expansion QMC method 19,01 is an improved variant of the so called Handscomb's technique. 22 It is based on importance sampling of terms of the power series expansion of $\exp (-\beta \hat{H})$, which for a finite system at finite $\beta$ can be carried out to all important orders, without introducing systematic errors. The current formulation of the method is described in Ref. 11, and has preyiously been applied to both static and dynamic properties of Heisenberg models in several different geometries 14.15 Here we briefly review how imaginary-time independent correlation functions are evaluated using this technique.

The Hamiltonian for an $N$-site periodic chain is first written as

$$
\hat{H}=-\frac{J}{2} \sum_{b=1}^{N}\left(\hat{H}_{1, b}-\hat{H}_{2, b}\right)+\frac{N J}{4},
$$

where the operators $\hat{H}_{1, b}$ and $\hat{H}_{2, b}$ are defined as

$$
\begin{aligned}
& \hat{H}_{1, b}=2\left(\frac{1}{4}-S_{b}^{z} S_{b+1}^{z}\right), \\
& \hat{H}_{2, b}=S_{b}^{+} S_{b+1}^{-}+S_{b}^{-} S_{b+1}^{+} .
\end{aligned}
$$

The partition function, $Z=\operatorname{Tr}\left\{\mathrm{e}^{-\beta \hat{H}}\right\}$, is transformed into a sum suitable for importance sampling techniques by expanding $\mathrm{e}^{-\beta \hat{H}}$ in a power series, and writing the trace as a sum over diagonal matrix elements in the standard basis $\{|\alpha\rangle\}=\left\{\left|S_{1}^{z}, \ldots, S_{N}^{z}\right\rangle\right\}$. This gives 


$$
Z=\sum_{\alpha} \sum_{n} \sum_{S_{n}} \frac{(-1)^{n_{2}}}{n !}\left(\frac{\beta}{2}\right)^{n}\left\langle\alpha\left|\prod_{l=1}^{n} \hat{H}_{a_{l}, b_{l}}\right| \alpha\right\rangle
$$

where $S_{n}$ denotes a sequence of index pairs defining the operator string $\prod_{l=1}^{n} \hat{H}_{a_{l}, b_{l}}$,

$$
S_{n}=\left[a_{1}, b_{1}\right]\left[a_{2}, b_{2}\right] \ldots\left[a_{n}, b_{n}\right], \quad a_{i} \in\{1,2\}, b_{i} \in\left\{1, \ldots, N_{b}\right\},
$$

and $n_{2}$ denotes the total number of index pairs (operators) $\left[a_{i}, b_{i}\right]$ with $a_{i}=2$. For even $N$ (or any $N$ for an open chain) $n_{2}$ is even for all non-zero terms in Eq. (24). All terms are then positive, and can be stochastically sampled using standard Monte Carlo techniques in the space of index sequences and basis states.

The simulation is carried out using two different elementary modifications of the index sequence, taking into account the constraints imposed by the fact that the operators defined in Eqs. (23) are allowed to operate only on antiferromagnetically aligned spin pairs, and that the operator product corresponding to $S_{n}$ must propagate the state $|\alpha\rangle$ onto itself. The power $n$ is changed by inserting or removing single diagonal operators $[1, b]$, and the number of spin flipping operators is changed by pair-substitutions $[1, b],[1, b] \leftrightarrow[2, b],[2, b]$ (the two operators selected for updating are typically not adjacent in the sequence). The grand canonical ensemble with fluctuating total magnetization, $m^{z}=\sum_{i} S_{i}^{z}$, can be studied for $T / J \gtrsim 0.08$ by also performing spin flips in the states. At lower temperatures the acceptance rate for such flips becomes very low, and it is then more convenient to study the canonical ensemble with $m^{z}=0$.

QMC calculations can access the dynamic structure factor only through the corresponding correlation function in imaginary time,

$$
C_{r_{1}, r_{2}}(\tau)=\left\langle S_{r_{1}}^{z}(\tau) S_{r_{2}}^{z}(0)\right\rangle .
$$

In the stochastic series expansion method, such a correlation function is estimated by measuring the correlations between states $|\alpha(p)\rangle=\left|S_{1}^{z}[p], \ldots, S_{N}^{z}[p]\right\rangle$ obtained by propagating $|\alpha\rangle$ in Eq. 24] with $p$ of the operators in the product:

$$
|\alpha(p)\rangle=\prod_{l=1}^{p} \hat{H}_{a_{l}, b_{l}}|\alpha\rangle, \quad|\alpha(0)\rangle=|\alpha\rangle .
$$

The expression for the imaginary-time dependent spin-spin correlation function is 10

$$
C_{r_{1}, r_{2}}(\tau)=\left\langle\sum_{m=0}^{n} \frac{\tau^{m}(\beta-\tau)^{n-m} n !}{\beta^{n}(n-m) ! m !} \bar{C}_{r_{1}, r_{2}}(m)\right\rangle
$$

where

$$
\bar{C}_{r_{1}, r_{2}}(m)=\frac{1}{n+1} \sum_{p=0}^{n} S_{r_{1}}^{z}[p] S_{r_{2}}^{z}[p+m]
$$

The periodicity of the propagated states imply that $S_{r}^{z}[p+n]=S_{r}^{z}[p]$. Note that any value of $\tau$ is accessible, in contrast to worldline methods where $\tau$ must be an integer multiple of the time-slice width used in the simulation.25 The corresponding static susceptibility $\chi_{r_{1}, r_{2}}$ (i.e., the real-space version of Eq. (8)), can be directly obtained by integrating over $\tau$ in (28). The result is 12

$$
\chi_{r_{1}, r_{2}}=\left\langle\frac{\beta}{n(n+1)}\left(\sum_{p=0}^{n-1} S_{r_{1}}^{z}[p]\right)\left(\sum_{p=0}^{n-1} S_{r_{2}}^{z}[p]\right)+\beta \frac{\bar{C}_{r_{1}, r_{2}}(0)}{n+1}\right\rangle .
$$

The relation between $C_{r_{1}, r_{2}}(\tau)$ and the dynamic structure factor defined in Eq. (5) is

$$
S_{k}(\tau)=\frac{1}{\pi} \int_{0}^{\infty} d \omega S(k, \omega) K(\omega, \tau)
$$

where $S_{k}(\tau)$ is the Fourier transform 


$$
S_{k}(\tau)=\frac{1}{N} \sum_{r_{1}, r_{2}} \mathrm{e}^{-i k\left(r_{2}-r_{1}\right)} C_{r_{1}, r_{2}}(\tau),
$$

the kernel is

$$
K(\omega, \tau)=\mathrm{e}^{-\tau \omega}+\mathrm{e}^{-(\beta-\tau) \omega},
$$

and $S(k,-\omega)=\mathrm{e}^{-\beta \omega} S(k, \omega)$. The anglytif continuation of the QMC data for $S_{k}(\tau)$, i.e., inversion of Eq. (31), is carried out using the Max-Ent method, 16,17 which we very briefly review here for completeness.

For a given momentum transfer $k, S(k, \omega)$ is paremetrized as a sum of $\delta$-functions at frequencies $\omega_{n}, n=1, \ldots N_{\omega}$,

$$
S(k, \omega)=\sum_{n=1}^{N_{\omega}} S_{n} \delta\left(\omega-\omega_{n}\right) .
$$

The "classic" variant of the Max-Ent method used here amounts to determining the coefficients $S_{n}$ that minimize the quantity

$$
Q=\chi^{2} / 2-\alpha E .
$$

Here $\chi^{2}$ is the deviation of the imaginary-time function $\bar{S}_{k}(\tau)$ corresponding to a particular set of spectral weights $\left\{S_{1}, \ldots, S_{n}\right\}$ in Eq. (34) from the QMC estimate $S_{k}(\tau)$ and its statistical error $\sigma(\tau)$, which is available for a discrete set of times $\tau_{1}, \ldots, \tau_{N_{\tau}}: 29$

$$
\chi^{2}=\sum_{i=1}^{N_{\tau}}\left[S_{q}\left(\tau_{i}\right)-\bar{S}_{q}\left(\tau_{i}\right)\right]^{2} / \sigma\left(\tau_{i}\right)^{2} .
$$

$E$ is the entropy, defined with respect to a "default model" $m(\omega)$. Using a default which is constant for $\omega>0$ and satisfies $m(-\omega)=\mathrm{e}^{-\beta \omega} m(\omega)$, the entropy is (with $S(k, \omega)$ assumed normalized to unity),

$$
E=-\sum_{i=1}^{N_{\omega}} S_{n} \ln \left(S_{n}\right) K\left(\omega_{n}, 0\right) .
$$

The parameter $\alpha$ in Eq. (35) is determined iteratively using a criterion derived using Bayesian logic, leading to the most probable $S(k, \omega)$ compatible with the QMC data and its errors, as discussed in detail in Ref. 17. Typically, on the order of $N_{\omega}=100-200$ frequencies are used in Eq. (34). The amplitudes $S_{n}$ then form a smooth curve representing the frequency dependence of $S(k, \omega)$.

\section{Comparisons with Exact Diagonalization}

Accuracies of calculations of dynamic quantities using the HTE and QMC methods are difficult to assess rigorously. Comparing results obtained in the two different ways provides for a good test. However, the results will never agree completely (In contrast, for static quantities the results agree perfectly in the regime where HTE performs well, as discussed in Sec. III-C.), and it is therefore useful to check the results against other calculations as well.

For a small system, all the eigenstates can be obtained exactly by numerically diagonalizing the Hamiltonian. Using the translational invariance and the conservation of the $z$-component of the spin, the Hamiltonian consists of blocks corresponding to all the combinations of the magnetization $m^{z}$ and the momentum $k$. For a 16-site system the largest blocks have 810 states, and can easily be diagonalized on a workstation. The next two appropriate sizes, $N=18$ and $N=20$, have largest-block sizes of 2704 and 9252 states, respectively, and could be studied with some more effort. Here we consider only $N=16$.

The exact dynamic structure factor of a finite system is a set of $\delta$-functions with positions given by the energies of the excited states, and amplitudes given by the squares of the corresponding matrix elements of the operator $S_{k}^{z}$. For a 16-site system, the number of contributing $\delta$-functions is very small at $T=0$, and it is not possible to carry out a meaningful comparison with the other methods. As the temperature is raised, the number of $\delta$ functions with significant weights increases rapidly, and a relatively smooth spectrum can be obtained by using some reasonable broadening of the individual $\delta$-functions. The results can of course not be expected to completely represent the thermodynamic limit, but at temperatures where the correlation length is much smaller than the system 
size meaningful comparisons should be possible. We here compare HTE, QMC, and exact diagonalization results for $S(k, \omega)$ at $k=\pi / 2$.

Fig. [4 shows results of all the methods at temperatures $T / J=1.0$ and 0.5 . We have chosen to represent the exact results as histograms with a bin width $\Delta_{\omega}=0.1 J$. The $n$th bin contains the integrated spectral weight in the frequency range $\left[(n-1) \Delta_{\omega}, n \Delta_{\omega}\right]$. On this frequency scale, the results still have structure due to the finite size, but nevertheless exhibit over-all shapes that the other results can be compared with. Indeed, the HTE spectra have shapes that very well match the histograms. The QMC + Max-Ent results are somewhat broader and have more rounded shapes (less asymmetric), as would also be expected, but still represent quite reasonable distributions of spectral weight. The QMC results were calculated for a system of $N=128$ sites. Results obtained for the same size, $N=16$, as the exact diagonalization, look very similar, which also indicates that finite size effects are small at these temperatures (for the momentum considered here). The relative statistical errors of the imaginary-time data used were in the range $10^{-4}-10^{-3}$, which is typical for all the QMC results discussed in this paper (the absolute error is typically in the fifth decimal place of the result).

The limit $\omega \rightarrow 0$ is of special interest, as it determines the spin-lattice relaxation rate. The results shown in Fig. A indicate that meaningful results can be obtained for this quantity. The differences between the HTE and QMC + Max-Ent results are typically 10-20\%. We cannot rigorously establish which calculation is more accurate in the $\omega \rightarrow 0$ limit, but based on the better agreement with the over-all shape between HTE and exact diagonalization in the whole frequency range, we expect HTE to be more accurate in the temperature regime where it performs well $(T / J \gtrsim 0.5)$. At lower temperatures only QMC + Max-Ent results are available, since exact diagonalization also does not provide accurate results below $T / J \lesssim 0.5$, especially for $\omega \rightarrow 0$. Based on high-temperature comparisons such as those presented here, we expect the error of the QMC + Max-Ent calculations at lower temperatures to be of the order $10-20 \%$.

\section{RESULTS}

The HTE method is best suited for studying the dynamics of $k$-integrated quantities. Apart from the results for $k=\pi / 2$ presented above, we therefore discuss HTE results mainly for momentum averages and the spin-lattice relaxation rate $1 / T_{1}$. With the QMC method we have calculated the imaginary-time correlation functions needed to obtain the full $S(k, \omega)$ for systems with up to $N=128$, down to temperatures $T / J=1 / 8$. Results for $1 / T_{1}$, as well as the transverse rate $1 / T_{2 G}$, were already presented in Ref. 15, where $1 / T_{1}$ was calculated for slightly larger systems by analytically continuing weighted imaginary-time correlation averages for space separations $r \leq 2$, corresponding to the model form factor discussed in Sec. II. Here $S(k, \omega)$ is first calculated for all momenta, and the momentum averaging is carried out after the analytic continuations. This method, though much more cumbersome and time consuming, should be more reliable for studying long-time tails such as those resulting from spin diffusion at high temperatures.

Below, in Sec. III-A, we first consider various aspects of the frequency and momentum dependence of the dynamic structure factor. In III-B we discuss the spin-lattice relaxation rate, and recent experimental results for $\mathrm{Sr}_{2} \mathrm{CuO}_{3}$ and $\mathrm{CuGeO}_{3}$. In III-C we discuss the scaling behavior of the dynamic susceptibility, and how it is affected by logarithmic corrections. We present explicit calculations of the logarithmic corrections to the temperature dependence of the staggered structure factor and the staggered susceptibility.

\section{A. The Dynamic Structure Factor}

Results for $S_{0}(\omega)$ (corresponding to a form factor $A(k)=1$ ), obtained using the HTE technique at different temperatures are shown in Fig. 5 . As discussed earlier, we expect these results to be reliable down to $T / J=0.5$. We find that at very high temperatures there is strong $\omega^{-\alpha}$ divergence which diminishes as $T$ is decreased, and spectral weight is transferred to a broad peak at $\omega \sim 1.5$. At $T=1$, a peak at $\omega \sim 1.5$ is evident together with an infrared peak, which is still strong at this temperature. However, at $T=0.5$ the diffusion peak is almost invisible and most of the spectral weight is concentrated at higher frequencies.

$S_{0}(\omega)$ calculated using QMC and Max-Ent analytic continuation is shown in Fig. 6, for temperatures down to $T / J=1 / 8$. Results for systems with $N=64$ and $N=128$ are compared, in order to assess effects of the system size. The agreement with the HTE results is quite good for $T / J=1.0$ and 0.5 . The diffusive $\omega \rightarrow 0$ peak at $T=1.0$ is somewhat higher and narrower in the HTE result, whereas it is somewhat more pronounced in the QMC results at $T / J=0.5$. At high temperatures, the $\omega \rightarrow 0$ peak height grows with the system size in a QMC calculation, since the diffusive contributions are cut-off at the momentum $k_{1}=2 \pi / N$ in a finite system. Considering the intrinsic 
difficulties of numerical analytic continuation, in particular of QMC data, the agreement between the HTE and QMC + Max-Ent results has to be considered quite satisfactory.

Apart from the $\omega \approx 0$ peak at high temperatures, the differences between the $N=64$ and $N=128$ results are likely mainly due to statistical fluctuations in the imaginary-time data, which are amplified in the real frequency spectra. The dominant peak at $\omega / J \approx 1.5$ is very pronounced at $\beta=8$, and does not exhibit much size dependence. The position of the peak can be understood on the hasis of the $T=0$ two spinon continuum. The Bethe ansatz solution gives an exact expression for the lower edge, $30 \omega_{\min }(k)=(\pi / 2) J \sin (k)$. The upper bound is known to be 21 $\omega_{\max }(k)=\pi J \sin (k / 2)$. Since the dominant spectral weight is concentrated at the lower edge, one can expect a maximum in the momentum average $S_{0}(\omega)$ at $\omega=\pi / 2$, arising from momenta $q \approx \pi / 2$, where the lower bound has the smallest $q$-dependence. This is indeed the position of the maximum seen in Fig. 6 at $\beta=8$.

A maximum at lower frequency also develops in $S_{0}(\omega)$ for $T<0.25 \mathrm{~J}$. It is due the gradual change from relaxational to propagating behavior for modes with momenta $3 \pi / 4 \lesssim k \leq \pi$. The peak sharpens and moves towards $\omega=0$ as the temperature is lowered (this trend is seen also in results for lower temperatures, not shown here). This maximum is expected from the scaling form for the dynamic susceptibility first derived by Schulzt $\left(\chi^{\prime \prime}(\omega) \sim \tanh (\omega / 2 T)_{\text {for }}\right.$ $T \ll J)$, and has also been discussed in the context of neutron scattering experiments on $\mathrm{Cu}_{(}\left(\mathrm{C}_{6} \mathrm{D}_{5} \mathrm{COO}\right)_{2} \cdot 3 \mathrm{D}_{2} \mathrm{O}$. 31

Fig. 7 shows the momentum dependence of $S(k, \omega \rightarrow 0)$ at several temperatures. It is clear that the low-frequency weight is strongly peaked near $k=0$ at $T / J=1$, but shifts to $k=\pi$ as the temperature is lowered. The strong increase as $k \rightarrow 0$ at high temperatures is clearly not captured completely in a small system, due to the discreteness of the momentum. Apart from this long-wavelength cut-off, there is no size dependence between $N=64$ and 128 within the fluctuations of the data. We have not explicitly calculated statistical errors of the results, but one can get an impression of their order from the (rather low) degree of non-smoothness of the curves. As discussed in Sec. II-C, there may be some systematical errors present as well, due to the unavoidable bias of the Max-Ent method used for the analytic continuation. In particular, this may be the case close to both $k=0$ and $k=\pi$, in the neighborhood of the points where low frequency weight first starts to appear (i.e. at temperatures where there is an intermediate momentum regime with vanishing low-frequency weight). In these regimes $S(k, \omega \rightarrow 0)$ may be over-estimated due to broadening effects, i.e., low frequency weight may be seen in Fig. 6 where in fact the actual modes only begin to have significant weight slightly above $\omega=0$. Away from these regimes, we expect systematic errors of at most $10-20 \%$, as discussed in Sec. II-C.

We now discuss the full dynamic structure factor in the temperature regime where there is the most significant shift in spectral weight from $k \approx 0$ to $k \approx \pi$. We present $N=128$ results for $T / J=1.0,0.5$, and 0.25 graphed in two different ways. First, in Fig. 8, we show 3D graphs with curves of $S(k, \omega)$ for each individual $k$. This clearly demonstrates how the narrow $k \approx 0$ peak present at $T / J=1$ is significantly reduced at $T / J=0.5$, where there is also a massive build-up of spectral weight at momenta close to $k=\pi$. The maximum at $k=\pi$ is not yet very pronounced at this temperature, however. At $T / J=0.25$ the $k=0$ peak has vanished almost completely, and the distribution of spectral weight starts to resemble what would be expected from the $T=0$ two-spinon continuum. Again, the lack of smoothness along momentum cross-sections gives an impression of the considerable amplification by analytic continuation of the very small statistical errors in the imaginary-time data. The concentration of the weight between the lower and upper bounds of the two-spinon spectrum is seen more clearly in the plots of Fig. 9. Here the intensity is represented by shades of gray in the $(k, \omega)$-plane, and the $T=0$ bounds are also shown. It is clear that there is very little weight above the upper bounds even at high temperatures, whereas there is significant weight below the lower bound.

One may still wonder how well the Max-Ent method captures the true temperature dependence of $S(k, \omega)$. In the previous low-temperature calculations by Deisz et al. 19 considerable weight was observed below the rigorous lower bound even at temperatures as low as $T / J=1 / 24$, and the expected concentration of weight at the lower edge was not well reproduced. Above we have shown that our high-temperature results agree well with HTE calculations. In order to further investigate the broadening effects due to Max-Ent analytic continuation, we have also carried out simulations for the system size and temperature considered by Deisz et al. $(N=64, \beta=24)$. Fig. 10 shows our result for $k=3 \pi / 4$, which can be compared with Fig. 9 of Ref. 19. Our result indeed peaks at the lower bound, and is significantly less broadened towards lower frequencies. This probably reflects a higher accuracy in the underlying imaginary-time data. The broadening that is present at $\beta=24$ may still be partly due to temperature effects, but is likely mainly Max-Ent induced. This kind of broadening should be a problem primarily in cases where the lower edge is sharp, i.e., at very low temperatures. It will be present to some extent also for temperatures and momenta where there is very little low-frequency weight, and, as already discussed above, may then lead to an over-estimation of $S(k, \omega \rightarrow 0)$. The broadening below the lowest bound seen in the results of Figs. 8 and 9 is considerably larger than in Fig. 10, and we therefore believe that it is mainly due to real temperature effects, with only minor distortions due to Max-Ent bias. 


\section{B. Spin-Lattice Relaxation Rate}

Next we discuss our calculations of the nuclear spin-lattice relaxation rates $1 / T_{1}$, for different hyperfine couplings parametrized by the ratio $R$ in Eq. (6).

The QMC results are obtained by averaging the low-frequency dynamic structure factor shown in Fig. 7 (for $N=128$ ). As already mentioned, this method differs from the approach previously used in Ref. 15, where the averaging was done for the short-distance imaginary-time correlation functions, and the analytic continuation carried out subsequently. With exact imaginary-time data, the two approaches would yield identical results, but in the presence of statistical errors the Max-Ent method will bias the outcome differently in the two approaches. As an illustration of this, results for the on-site dynamic structure factor $S_{0}(\omega)$ obtained using the two different orders of averaging and analytic continuation are shown in Fig. 11. It is clear that the analytic continuation of the on-site function $S_{0}(\tau)$ misses much of the diffusive behavior for $\omega \rightarrow 0$ at the temperatures where these contributions are the most important. Based on the previous comparisons with HTE results at high temperatures, we believe that the approach of continuing $S(k, \omega)$ individually for each $k$ before averaging is more accurate. This can also be understood on grounds that the frequency dependence of $S(k, \omega)$, for a given $k$, has less structure than the momentum average, and is therefore easier to reproduce with a high-entropy curve (which is favored by the Max-Ent method).

The HTE + recursion method is applied to the short-distance correlations, according to Eq. (7). The zero frequency limit will produce a divergent $1 / T_{1}$ when infrared singularities are present. However, the very low-frequency, longwavelength limit of our results may not be accurate and should be viewed with some caution as the method is based on a short-time expansion, involving only finite spin clusters. We have therefore chosen not to focus on the exact form of the $\omega \rightarrow 0, q \rightarrow 0$ behavior. For calculating $1 / T_{1}$ we set the nuclear resonance frequency to $0.01 \mathrm{~J}$. In a calculation using QMC + Max-Ent, the divergence is cut off due to the finite size, and may also be rounded due to resolution effects. In real materials, several energy scales including anisotropy and coupling between chains serve to determine the cutoff frequency.

Results for $1 / T_{1}$ from the recursion method are shown for several different values of $R$ in Fig. 12, along with the $\mathrm{QMC}+$ Max-Ent results for $R=0$ and $R=-0.5$. The agreement between the HTE and QMC calculations is quite good for $T / J \geq 0.5$, and gives us confidence in the QMC data at lower temperatures. The main difference from the previous 15 QMC + Max-Ent results is that the diffusive contributions for $R=0$ at $T \gtrsim 0.5$ are stronger, as already discussed. At low temperatures the present results are $\approx 20$ percent lower than the previous results, both for $R=0$ and $R=-0.5$. It is likely that the present results at low temperatures are still somewhat too high (likely $10-20$ percent), due to the broadening effect of the max-ent method discussed above (see also Ref. 22, for a comparison of results for the ratio $T_{2 G} / \sqrt{T} T_{1}$ ).

Sachder calculated $1 / T_{1}$ using the scaling form of $S(k, \omega)$ obtained from bosonization and conformal field theory 83.8 This gives a temperature independent rate, which however is expected to be modified by logarithmic corrections to yield a logarithmic divergence as $T \rightarrow 0$. $\mathrm{S}$ This is clearly in qualitative agreement with the above results for $R=-0.5$ (where the diffusive contributions neglected in the theory are filtered out), but the accuracy is not high enough to extract a form for the low-temperature behavior. We have previously discussed other effects of logarithmic correction on the NMR relaxation rates.22 In particular, it can be noted that the ratio $T_{2 G} / \sqrt{T} T_{1}$ is modified from the universal temperature-independent form, approaching the universal value with infinite slope as $T \rightarrow 0$ (in a manner similar to the bulk susceptibility, as discussed in 34 ).

We now briefly discuss recent experimental results. Takigawa et al. measured both $1 / T_{1}$ and $1 / T_{2}$ in $\mathrm{Sr}_{2} \mathrm{CuO}_{3}$, 2 for which an exchange $J \approx 2000 \mathrm{~K}$ had been previously deduced from susceptibility measurements. 1 The hyperfine form factor could be accurately extracted 26 using an impurity effect on the NMR line shape predicted by Eggert and Affleck.27 Hence, there are no free parameters, and non-ambiguous comparisons with model calculations can be carried out. Takigawa et al. concluded that the agreement with the $1 / T_{2 G}$ calculation of Ref. 15 was good. For $1 / T_{1}$ the lowest temperature studied in Ref. 15 corresponds to $\approx 300 \mathrm{~K}$, which was the highest temperature studied experimentally. At this temperature, the previous QMC + Max-Ent result was $\approx 40 \%$ higher than the experimental value. As noted above, our improved method of calculating $1 / T_{1}$ gives a value $\approx 20 \%$ lower than before, and hence this discrepancy is now largely reconciled (the remaining differences can likely be explained by uncertainties in the experimental values of $J$ and the hyperfine couplings, and by remaining errors in the numerical result). As discussed above and in Ref. 22, also the slight temperature dependence observed for $T_{2 G} / \sqrt{T} T_{1}$ can be explained theoretically, and is largely due to logarithmic corrections. Hence, all the analytical and numerical results are now in good agreement with the experiments, indicating that $\mathrm{Sr}_{2} \mathrm{CuO}_{3}$ indeed is an almost perfect realization of the spin-half Heisenberg chain with only nearest-neighbor interactions.

We also note that some contributions from diffusive professes, signaled by a dependence of $1 / T_{1}$ on $\omega_{N}$ (i.e., the external field strength) were explicitly observed in $\mathrm{Sr}_{2} \mathrm{CuO}_{3}$ 目 It would clearly be interesting to study this material also at higher temperatures, where our numerical results indicate that a considerably stronger diffusive behavior should 
be observed.

Another quasi-1D compound which has been studied very actively recently is $\mathrm{CuGeO}_{3}$, which undergoes a spinPeierls $(\mathrm{SP})$ transition at $T \approx 14 \mathrm{~K} .6$ This material has a stronger coupling between the chains than $\mathrm{Sr}_{2} \mathrm{CuO}_{3}$, and also is expected to have a non-negligible next-nearest-neighbor interaction $J_{2} 351 / T_{1}$ exhibits a strong, almost linear, decrease with decreasing temperature, with a reduction by almost a factor of 5 between $T \approx J$ and $T \approx J / 5$ (If $J_{2}$ indeed is significant, $J$ should here be considered an effective coupling constant).36.37 Itoh et al. argued that the transferred hyperfine term is small, 36 and hence $R \approx 0$ in our notation. Our results shown in Fig. 12 then indicate a reduction by a factor of 2 in the aboye temperature regime, which is significantly different from the experiment. On the other hand, Fagot-Revurat et al. 37 argued that there is a significant transferred hyperfine coupling, and they were able to obtain a reasonable fit to the previous $1 / T_{1}$ results for $R \approx 0.25 .5$ Notice that this value of $R$ leads to a strong enhancement of contributions from diffusive, $k \approx 0$, modes, and as a result, a stronger temperature dependence of $1 / T_{1}$ (as compared with, e.g., the $R=0$ case). Numerical calculations for the NMR rates including $J_{2}$, inter-chain couplings, and dynamic phonons have yet to be carried out, and would clearly be very useful for determining the importance of additional interactions beyond the standard Heisenberg chain in a realistic model of $\mathrm{CuGeO}_{3}$. The fact that the temperature dependence of $1 / T_{1}$ above the SP transition extrapolates to a positive value at $T=036.37$ indicates that the frustrating $J_{2}$ coupling by itself is not sufficient for opening a gap, but it may clearly contribute to stabilizing the dimerized state.

\section{Scaling Behavior}

In this section we discuss the low-temperature scaling behavior of the staggered susceptibility. At a $T=0$ quantumcritical point with $z=1$, conventional quantum critical scaling implies that the dynamic staggered susceptibility has the scaling form 38

$$
\chi(q, \omega)=\frac{a}{T^{2-\eta}} \phi\left(\frac{c q}{k_{B} T}, \frac{\omega}{k_{B} T}\right),
$$

where $a$ is a non-universal number and $\phi(x, y)$ is a universal complex function of both arguments and $q$ measures deviations from the antiferromagnetic wave-vector; $q=\pi-k$. This in turn also implies that near the antiferromagnetic wave-vector, the equal-time structure factor $S(q)$ and the antiferromagnetic susceptibility, $\chi(q)$ have scaling forms

$$
\begin{aligned}
& S(q) / S(0)=f_{1}(c q / T), \\
& \chi(q) / \chi(0)=f_{2}(c q / T) .
\end{aligned}
$$

However, for the Heisenberg chain these scaling relations are violated by logarithmic factors ${ }^{\text {which }}$ are produced by marginally irrelevant operators describing interaction between left- and right-moving currents 34 . The failure of scaling for $\chi(q)$ was discussed earlier, and a way to take the logarithmic corrections into account analytically was proposed.22 Here, in Fig. 13, we show the scaling plot for $S(q)$. Substantial systematic deviations from scaling in $q / T$ are apparent even at the lowest temperatures accessible to us. We also show results for the quantity $S^{\prime}(q)$, obtained by subtracting the ferromagnetic (uniform) component of the spin-spin correlation function from the numerical data for $S_{r}(0)$ before Fourier transforming to momentum space. The uniform term is given by $-\left[T /(2 \sinh (\pi T r / c)]^{-2}\right.$, and contains no adjustable parameters. $S^{\prime}(q)$ then contains fluctuations with momenta around the antiferromagnetic wave-number $\pi$ only, i.e. with $q \sim 0$. However, $S^{\prime}(q) / S^{\prime}(0)$ also does not exhibit scaling in $q / T$, demonstrating the importance of subleading non-universal corrections. Unlike what is the case for the static susceptibility, 22 which is dominated by low-frequency fluctuations, these deviations cannot be explained by taking only long-distance logarithmic corrections into account.

Turning now to scaling in $\omega / T$, we discuss the scaling amplitudes at $q=0$, i.e. the staggered structure factor and the staggered susceptibility. Including the leading logarithmic corrections, the sum rules (9) give the low-temperature forms

$$
\begin{aligned}
& S(q=0)=D_{s}\left[\ln \left(T_{s} / T\right)\right]^{3 / 2}, \\
& \chi(q=0)=D_{\chi} T^{-1}\left[\ln \left(T_{\chi} / T\right)\right]^{1 / 2} .
\end{aligned}
$$

In Fig. 14 these quantities are graphed in such a way that the behavior vs. $\ln (J / T)$ should be linear if the above asymptotic forms hold. The HTE results shown are from several different differential approximants. The agreement between the HTE and the QMC data is quite good down to $\beta \approx 6-8$, where the HTE approximants start to deviate from each other and from the QMC results, indicating that the HTE method becomes unreliable. Linear behavior 
consistent with Eqs. (40) is indeed observed in the QMC data for $J / T \gtrsim 4$. Fitting lines to the results in this regime gives $D_{s}=0.094 \pm 0.001, D_{\chi}=0.32 \pm 0.01, T_{s}=18.3 \pm 0.5$, and $T_{\chi}=5.9 \pm 0.2$.

The difference in $T_{s}$ and $T_{\chi}$ may be due to divergent contributions to $S(0)$ from short distances, careful treatment of which would require a separate cutoff. Another important reason is the ferromagnetic contribution to the spin-spin correlation function, already discussed above. This contributes to both $S(0)$ and $\chi(0)$. In Fig. 14 we also show results for the quantities $S^{\prime}(0)$ and $\chi^{\prime}(0)$, obtained by subtracting the ferromagnetic contributions from $S(0)$ and $\chi(0)$. The parameters obtained in this case are $D_{s}^{\prime}=0.106 \pm 0.001, D_{\chi}^{\prime}=0.34 \pm 0.01, T_{s}^{\prime}=5.1 \pm 0.2$, and $T_{\chi}^{\prime}=3.9 \pm 0.3$. The high-energy cutoffs $T^{\prime}$ obtained in this way are now much closer to each other, and also close to the value $T_{0}=4.5$ obtained in Ref. 22 by fitting a scaling form to the real-space correlation function at $\beta=32$. Within the accuracy of the numerical procedures (which apart from statistical errors also include effects from non-asymptotic contributions) they can be considered equal. We note that these parameters still should be viewed as 'effective' or temperaturedependent, and their asymptotic zero temperature yalues may only be reached at $T / J<0.01$ as is the case for the uniform susceptibility 34 and the correlation length 39 .

The temperature dependence of $\chi(q=0)$ is important in the context of systems of weakly coupled spin chains, where it determines the critical temperature for ordering into a three-dimensional Neel state 40 A recent calculation for $\mathrm{KCuF}_{3}$ by Schulz, 40 using our numerical data for $\chi(q=0)$, is in good agreement with experimental results.

\section{CONCLUSIONS}

In this paper, we have used several numerical methods to study the dynamics of the spin- $1 / 2$ Heisenberg chain at intermediate temperatures (above and below $J$ ). In order to obtain the dynamic structure factor, we have combined high temperature expansions for the frequency moments with the recursion method, and quantum Monte Carlo simulations with the maximum entropy method. In some cases, the results of these methods have been compared with exact results for a system with 16 spins, as an additional check.

We find that at high temperatures the HTE + recursion method works very well even for dynamic quantities. Using the first six continued fraction coefficients for the relaxation function, we have obtained dynamic susceptibilities at various temperatures down to $T / J=0.5$. The calculated frequency-dependence of the structure factor at $k=\pi / 2$ agrees remarkably well with the exact results on finite systems. This method is also sensitive to infrared singularities. Already at the level of the continued fractions themselves, that is before any numerical extrapolation is done, the presence or absence of singularities can be detected. We found that the behavior of the continued fractions for the local ( $k$-integrated) susceptibilities changes qualitatively depending on whether the hyperfine couplings are vanishing or non-vanishing at $k=0$. However, when infrared singularities are present, their exact forms may not be fully captured by these methods. Since the diffusion-related singularities are most robust at infinite $T$, where they have been investigated in the past with higher number of moments,23 we did not focus on this issue here. In our study, the HTE with the recursion method became unreliable below $T / J=0.5$ because the extrapolations for the higher moments became unstable. By extending the series for the frequency moments, it may be possible to reach still lower temperatures also for dynamic quantities.

The QMC results for the static quantities agree perfectly with the high temperature expansions down to $T / J \approx$ $0.1-0.2$, below which the HTE results become unreliable. Results obtained for the dynamic quantities with the QMC + Max-Ent method are in satisfactory (generally within 10-20 percent) agreement with HTE results above $T / J=0.5$ and, we believe, should have similar reliability down to much lower temperatures. For calculating local quantities such as $1 / T_{1}$, and incorporating as much as possible the effects of infrared singularities from certain $k$-regions, it appears to be better to carry out the analytic continuation for all individual momenta separately before performing the momentum sum, rather than carrying out the analytic continuation for momentum-integrated quantities.

We have also presented several new results for the Heisenberg chain. These include quantitative estimates for the logarithmic temperature dependence of the static staggered susceptibility and structure factor, improved estimates for the temperature dependence of the spin-lattice relaxation rate with various choices of hyperfine couplings, as well as the full dynamic structure factor at intermediate temperatures. Our results clearly show the shift in the low frequency spectral weight from the diffusive modes near $k=0$ at high temperatures to the antiferromagnetic modes near $k=\pi$ at low temperatures. At low temperatures we observed the development of spectral features that can be associated with the two-spinon continuum. Overall, our results at the lowest temperatures are in good agreement with with general expectation of these quantities from various analytical studies. Our results also allow us to reconcile the measurements of the spin-lattice relaxation rates in $\mathrm{Sr}_{2} \mathrm{CuO}_{3}$ with theoretical and numerical calculations for the Heisenberg model.

After completing this work, we became aware of a recent paper by Fabricius, Löw and Stolze,11 discussing exact diagonalization results for $S(k, \omega)$ of chains with up to 16 sites. We have here used such short-chain calculations 
mainly as a test of the HTE and QMC + Max-Ent methods in some regimes. Fabricius et al 1 found no signs of spin diffusion. Our results indicate that 16 sites is not large enough in general, in particular not for addressing the intricate problem of spin diffusion. As can be inferred from Fig. 7, the momentum cut-off $k_{1}=\pi / 8$ for a 16-site chain prohibits access to most of the long-wavelength regime where we see signs of diffusive behavior. In the limit $T \rightarrow 0$ there is most likely no spin diffusion, as discussed by Sachdev, 1 and also supported by the numerical results of Ref. 15 and our present calculations. We stress again that our study is also not accurate enough to resolve the exact form of $S(k, \omega)$ in the $k \rightarrow 0, \omega \rightarrow 0$ limit. We have strong evidence of a sharp peak at high temperatures, but cannot determine whether or not it is truly divergent (i.e., whether the long-time behavior of the spin-spin correlations is of the standard $1 \mathrm{D}$ diffusive form $\sim t^{-1 / 2}$, or, perhaps, is anomalous). In view of the absence of diffusion in the limit $T \rightarrow 0$, a sharp $\omega \rightarrow 0$ peak in $S_{0}(\omega)$ at high $T$, diverging as $T \rightarrow \infty$, is perhaps the most likely scenario. In any case, as we have discussed above, a sharp maximum should have detectable effects on, e.g., the spin lattice relaxation rate in real materials.

We would like to thank M. Horvatic, S. Sachdev, D. Scalapino, H. Schulz, and M. Takigawa for stimulating communication. This work was supported by NSF under Grants DMR-9318537 and DMR-9520776, and by the Campus Laboratory Collaboration of the University of California. The QMC calculations were carried out at the Supercomputations Research Institute at Florida State University.

a On leave from Institute for High Pressure Physics, Russian Academy of Sciences, Troitsk 142092, Moscow Region, Russia.

$b$ Present address: Department of Physics, University of Florida, P. O. Box 118440, Gainesville, Florida 32611.

${ }^{c}$ Present address: Department of Physics, University of Illinois at Urbana-Champaign, 1110 West Green Street, Urbana, Illinois 61801.

${ }^{1}$ T. Ami et al., Phys. Rev. B 51, 5994 (1995); N. Motoyama, H. Eisaki, and S. Uchida, Phys. Rev. Lett, 76, 3212 (1996).

${ }^{2}$ M. Takigawa, N. Motoyama, H. Eisaki and S. Uchida, Phys. Rev. Lett. 764612 (1996).

${ }^{3}$ K. Ishida et al., J. Phys. Soc. Jpn., 63, 3222 (1994).

${ }^{4}$ M. Greven et al., Phys. Rev. Lett. 72, 1096 (1994).

${ }^{5}$ D. A. Tennant et al., Phys. Rev. Lett. 70, 403 (1993).

${ }^{6}$ M. Hase, I. Terasaki, and K. Uchinokura, Phys. Rev. Lett. 70, 3651 (1993).

${ }^{7}$ H. J. Schulz, Phys. Rev. B 346372 (1986).

${ }^{8}$ S. Sachdev, Phys. Rev B 50, 13006 (1994).

${ }^{9}$ V. S. Viswanath and G. Müller, Recursion method - Application to Many-Body Dynamics, Lecture Notes in Physics, Vol. 23 (Springer-Verlag, New York 1994).

${ }^{10}$ A. W. Sandvik and J. Kurkijärvi, Phys. Rev. B 435950 (1991); A. W. Sandvik, J. Phys. A 25, 3667 (1992).

${ }^{11}$ A. W. Sandvik, in Strongly Correlated Magnetic and Superconducting Systems, Proceedings of the 1996 El Escorial Summer School, edited by G. Sierra and M.-A. Martin Delgado (Springer-Verlag, to be published).

12 D. C. Handscomb, Proc. Cambridge Philos. Soc. 58, 594 (1962); 60, 115 (1964); D. H. Lee, J. D. Joannopoulos, and J. W. Negele, Phys. Rev. B 30, 1599 (1984).

${ }^{13}$ N. Elstner et al., Phys. Rev. Lett. 75, 938 (1995).

${ }^{14}$ A. W. Sandvik and D. J. Scalapino, Phys. Rev. Lett. 72, 2777 (1994); Phys. Rev. B 51, 9403 (1995); Phys. Rev. B 53, R526 (1996); A. W. Sandvik, A. V. Chubukov, and S. Sachdev, Phys. Rev. B 51, 16483 (1995).; A. W. Sandvik, E. Dagotto, and D. J. Scalapino, Phys. Rev. B 53 R2934 (1996).

${ }^{15}$ A. W. Sandvik, Phys. Rev. B 52 R9831 (1995).

${ }^{16}$ R. N. Silver, D. S. Sivia, and J. E. Gubernatis, Phys. Rev. B 41, 2380 (1990); J. E. Gubernatis, M. Jarrell, R. N. Silver, and D. S. Sivia, ibid., B 44, 6011 (1991).

${ }^{17}$ M. Jarrell and J. E. Gubernatis, Phys. Rep. 269, 133 (1996).

18 J. Deisz, M. Jarrell, and D. L. Cox, Phys. Rev. B 42, 4869 (1990).

19 J. Deisz, M. Jarrell, and D. L. Cox, ibid, 48, 10227 (1993).

20 T. Moriya, Prog. Theor. Phys. 28, 371 (1962).

${ }^{21}$ G Müller et al. Phys. Rev. B 24, 1429 (1981); A. H. Bougourzi, M. Couture and M. Kacir,Phys. Rev. B 54, R12669 (1996); M. Karbach, G. Muller and A. H. Bougourzi, cond-mat/9606068.

${ }^{22}$ O. A. Starykh, R. R. P. Singh, and A. W. Sandvik, Phys. Rev. Lett. 78, 539 (1997).

${ }^{23}$ For a recent calculation of these moments at infinite $T$ see M. Böhm and H. Leschke, J. Phys. A 25, 1043 (1992).

${ }^{24}$ G. A. Baker et al. in Phase Transitions and Critical Phenomena Vol. 3, edited by C. Domb and M. S. Green (Academic, London 1974).

${ }^{25}$ J. E. Hirsch, R. L. Sugar, D. J. Scalapino and R. Blankenbecler, Phys. Rev. B 26, 5033 (1982). 
${ }^{26}$ M. Takigawa, N. Motoyama, H. Eisaki and S. Uchida, preprint (1996).

${ }^{27}$ S. Eggert and I. Affleck, Phys. Rev. Lett. 75, 934 (1995).

${ }^{28}$ P. C. Hohenberg and W. F. Brinkman, Phys. Rev. B 10, 128 (1974).

${ }^{29}$ In some cases it may be necessary to use the full covariance matrix in Eq. (36), instead of just its diagonal elements. 16 . 17

However, for the problem under consideration here, we find no significant differences between the two approaches.

${ }^{30}$ J. des Cloiseaux and J. J. Pearson, Phys. Rev. 128, 2131 (1962).

${ }^{31}$ D. C. Dender et al., Bull. Am. Phys. Soc., F9-3 (March 1996).

${ }^{32}$ D. A. Tennant, R. A. Cowley, S. Nagler and A. M. Tsvelik, Phys. Rev. B 5213368 (1995).

${ }^{33}$ S. Sachdev, T. Senthil, and R. Shankar, Phys. Rev. B 50, 258 (1994).

${ }^{34}$ S. Eggert, I. Affleck and M. Takahashi, Phys. Rev. Lett. 73, 332 (1994).

35 J. Riera and A. Dobry, Phys. Rev B 51, 16098 (1995); G. Castilla, S. Chakravarty, and V. J. Emery, Phys. Rev. Lett. 75, 1823 (1995).

${ }^{36}$ M. Itoh, M. Sugahara, T. Yamauchi, and Y. Ueda, Phys. Rev. B 54, R9631 (1996).

${ }^{37}$ Y. Fagot-Revurat et al., Phys. Rev. B (in press).

${ }^{38}$ A. Chubukov, S. Sachdev and J. Ye, Phys. Rev. B 49, 11919 (1994).

${ }^{39}$ K. Nomura and M. Yamada, Phys. Rev. B 43, 8217 (1991).

${ }^{40}$ H. J. Schulz, Phys. Rev Lett 77. 2790 (1996).

${ }^{41}$ K. Fabricius, U. Löw, and J. Stolze, preprint, cond-mat/9611077.

FIG. 1. Continued fraction coefficients, $\Delta_{k}$ for $S_{R}(\omega)$ as a function of $\beta$ for $R=0$ (lower panel) and as a function of the index $k$ for $R=0$ and -0.5 at $\beta=0$ (upper panel).

FIG. 2. Temperature dependence of the parameters of the fit for two values of $R$.

FIG. 3. Comparison of the real and model $\Delta$-sequences for two values of $R$ at different temperatures. Each panel shows $\Delta_{3}$ (bottom), $\Delta_{4}$ (middle), and $\Delta_{5}$ (top).

FIG. 4. Comparison of exact $N=16$ (histograms), HTE (solid curves) and N=128 QMC + Max-Ent (dashed curves) results for the dynamic structure factor at $k=\pi / 2$.

FIG. 5. The dynamic structure factor $S_{R}(\omega)$ with $R=0$ calculated using the HTE method at various temperatures.

FIG. 6. The dynamic structure factor averaged with a constant form factor calculated using QMC and Max-Ent analytic continuation at different temperatures. Dashed and solid lines are results for system sizes $N=64$ and 128 , respectively.

FIG. 7. The low-frequency limit of the dynamic structure factor vs. the momentum. Solid and open circles are for $N=64$ and 128 , respectively.

FIG. 8. QMC + Max-Ent results for the full dynamic structure factor $S(k, \omega)$ at three different temperatures for $N=128$. The maxima of the vertical scales are $3.01(T / J=1.0), 1.49(T / J=0.5)$, and $1.86(T / J=0.25)$.

FIG. 9. QMC + Max-Ent results for the dynamic structure factor $S(k, \omega)$ at $T / J=1.0,0.5$, and 0.25 , with shades of grey representing the intensity in the $(\omega, q)$ plane. The curves indicate the lower and upper bounds at $T=0$. Note that for $T=1.0$ and $0.5, S(k, \omega)$ is sharply peaked at $k \rightarrow 0, \omega \rightarrow 0$.

FIG. 10. QMC + Max-Ent results for the dynamic structure factor at $k=3 \pi / 4$, calculated for a 64 -site system at inverse temperature $\beta=24$. The dashed line is the $T=0$ Bethe ansatz lower edge.

FIG. 11. Comparisons of $S_{0}(\omega)$ calculated by averaging before (dashed curves) and after (solid curves) analytic continuation. 
FIG. 12. Results for NMR relaxation rates $1 / T_{1}$ for various values of the hyperfine parameter $R$, calculated using HTE and the QMC and Max-Ent methods.

FIG. 13. The equal-time structure factor $S(q)$ obtained from HTE and QMC, graphed so that the data would collapse onto a single curve if scaling in $q / T$ holds. The QMC results for $\beta \leq 8$ were calculated in the grand canonical ensemble for $N=256$, and those for $\beta \geq 16$ in the canonical ensemble for $N=1024$.

FIG. 14. The $T$-dependence of $S(k=\pi)$ and $\chi(k=\pi)$ obtained from HTE and QMC, graphed so that the predicted forms (40) give linear behavior. The dashed curves are several different HTE approximant, and the solid circles with error bars are the QMC results. The solid lines are fits to the QMC data for $T / J<0.25$. The open circles and dotted lines are results and fits after subtraction of the ferromagnetic contribution to the spin-spin correlation function. 

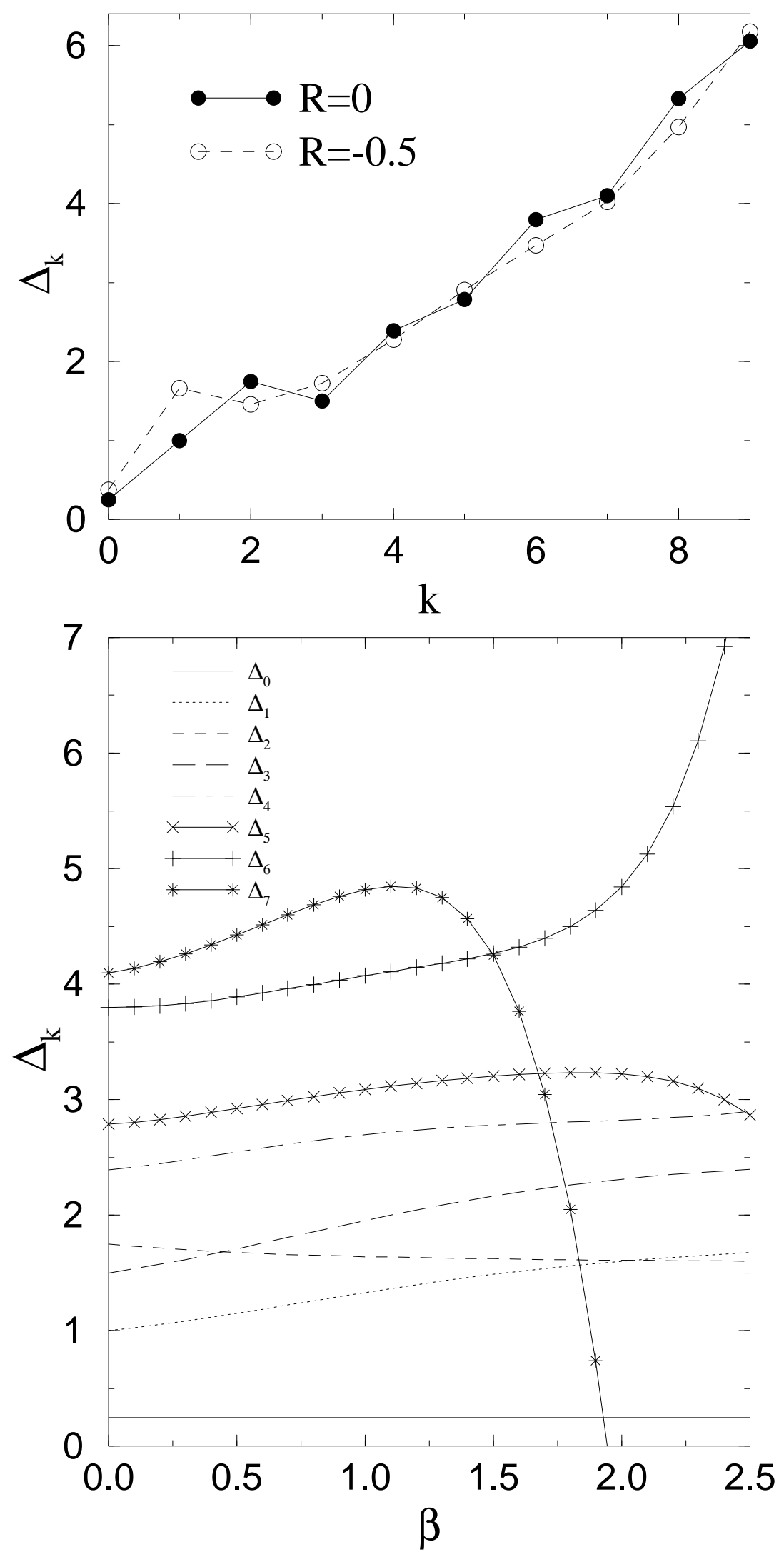

FIG. 1. O. A. Starykh, A. W. Sandvik, and R. R. P. Singh 


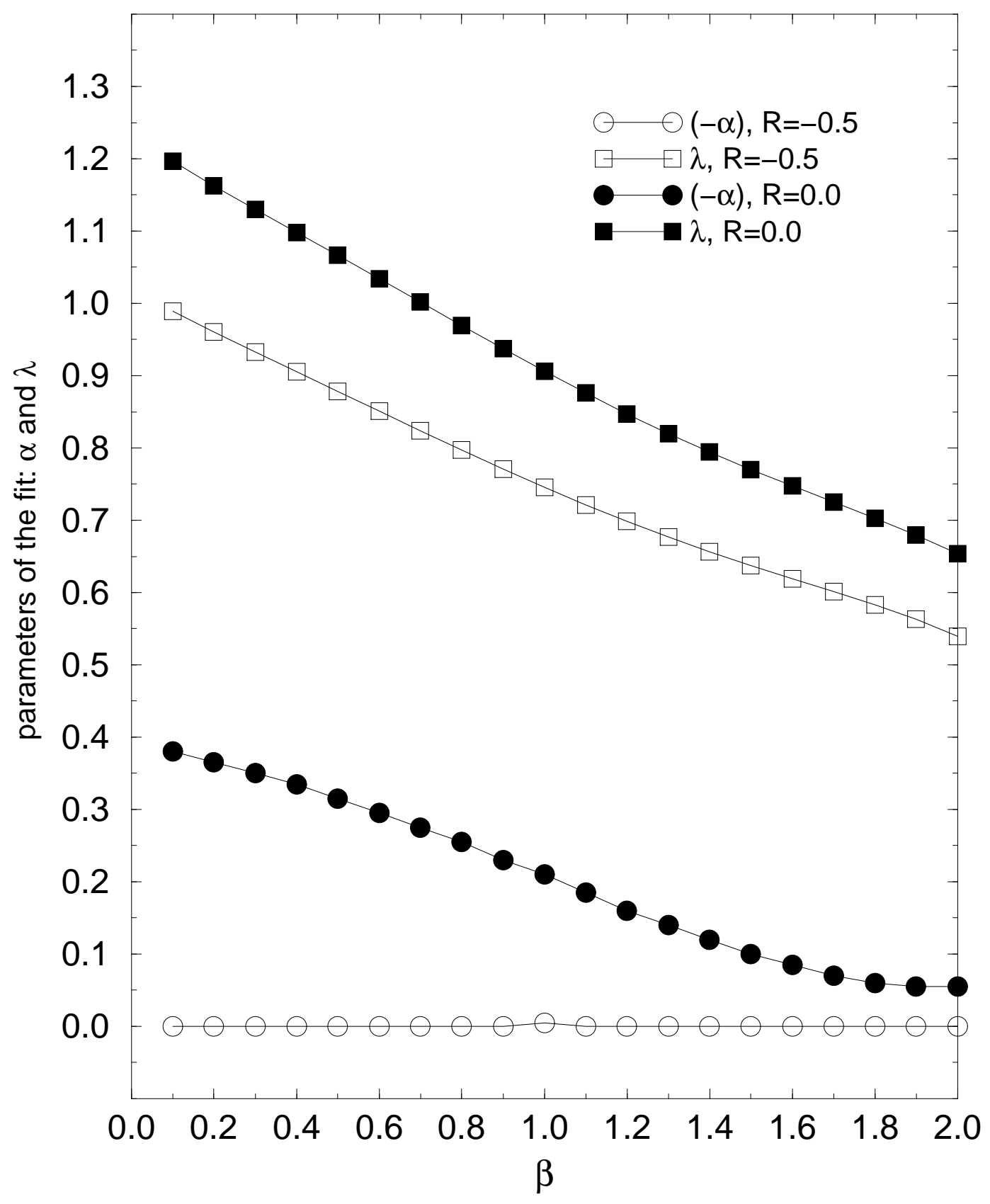

FIG. 2. O. A. Starykh, A. W. Sandvik, and R. R. P. Singh 

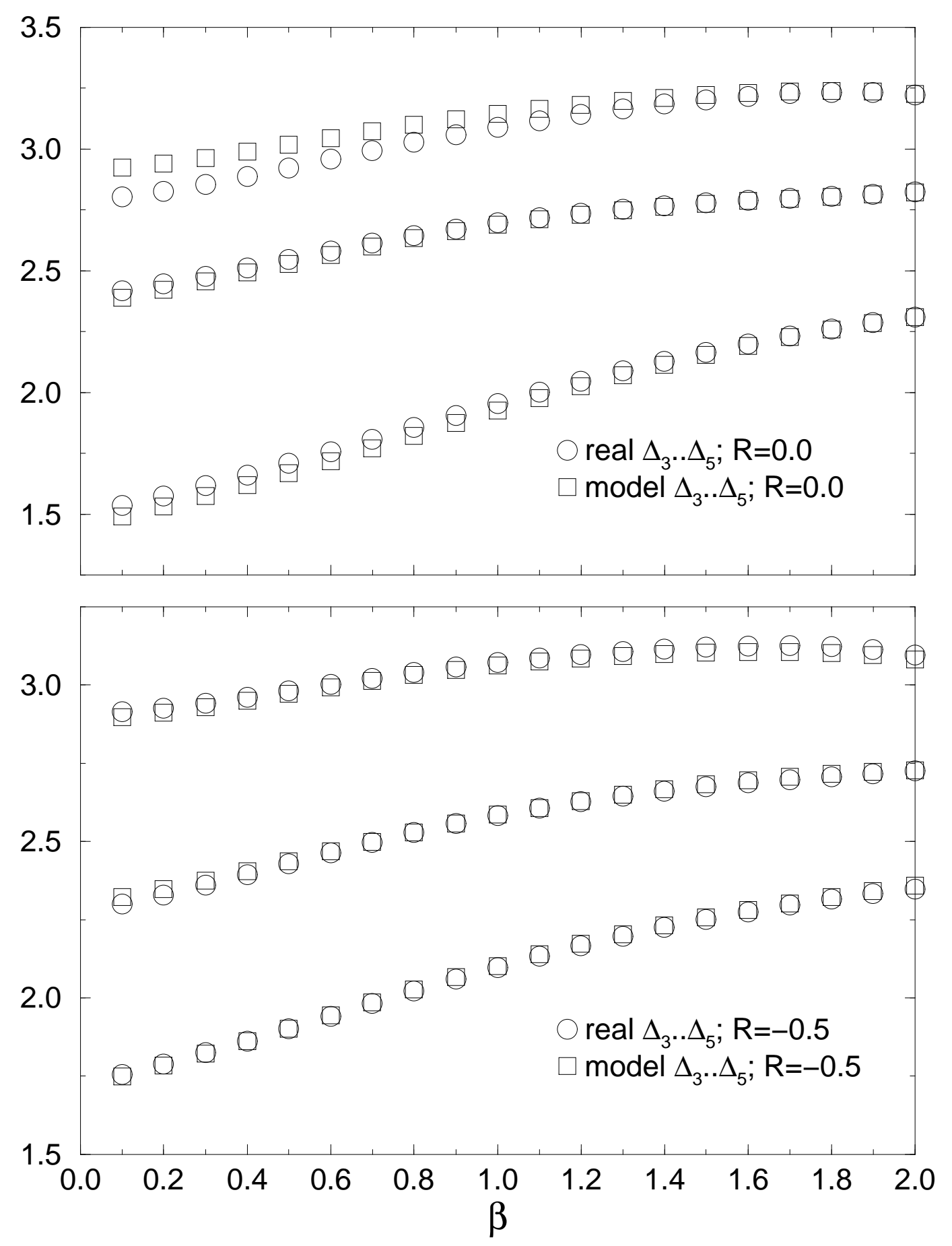

FIG. 3. O. A. Starykh, A. W. Sandvik, and R. R. P. Singh 


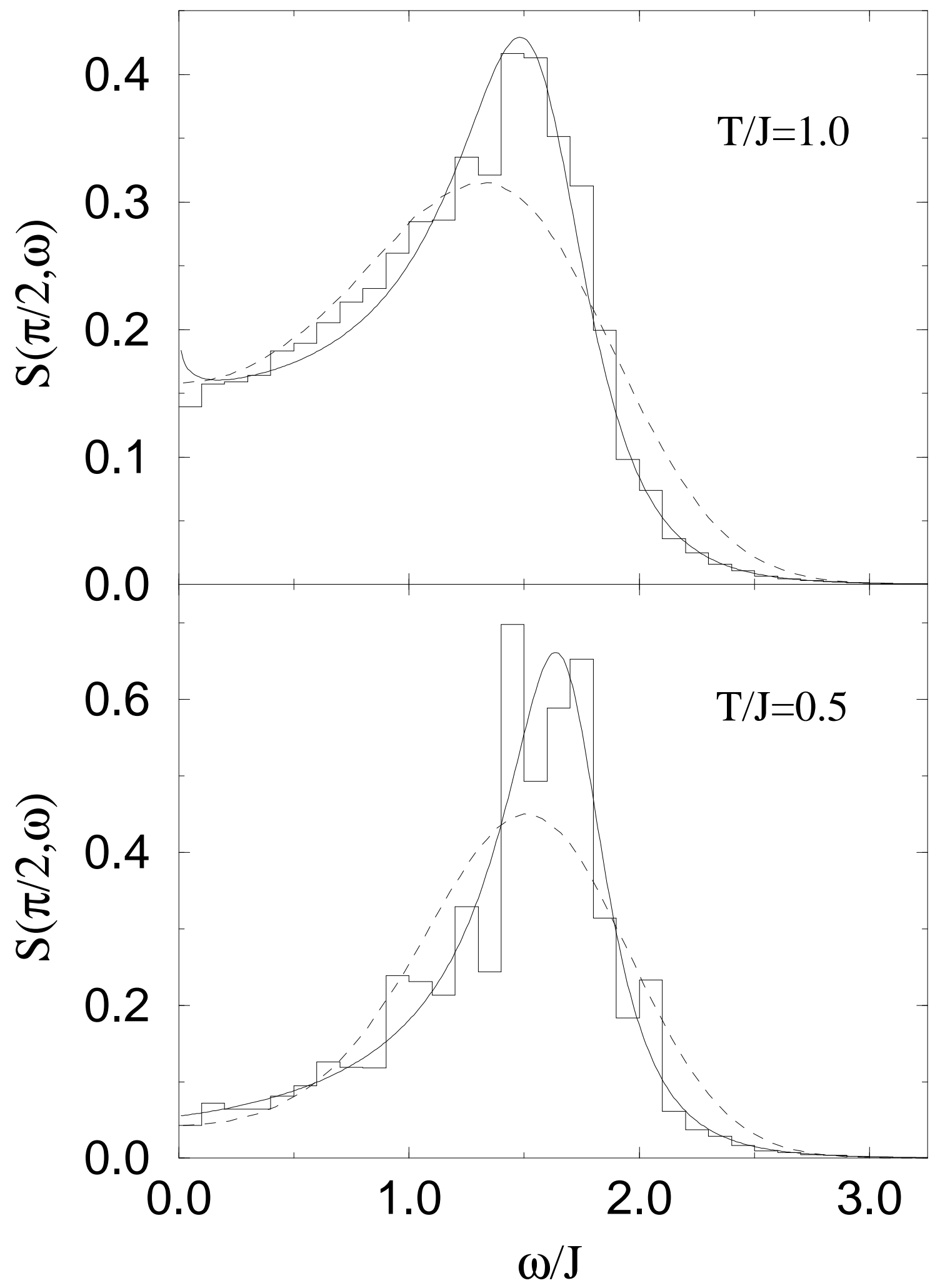

FIG. 4. O. A. Starykh, A. W. Sandvik, and R. R. P. Singh 


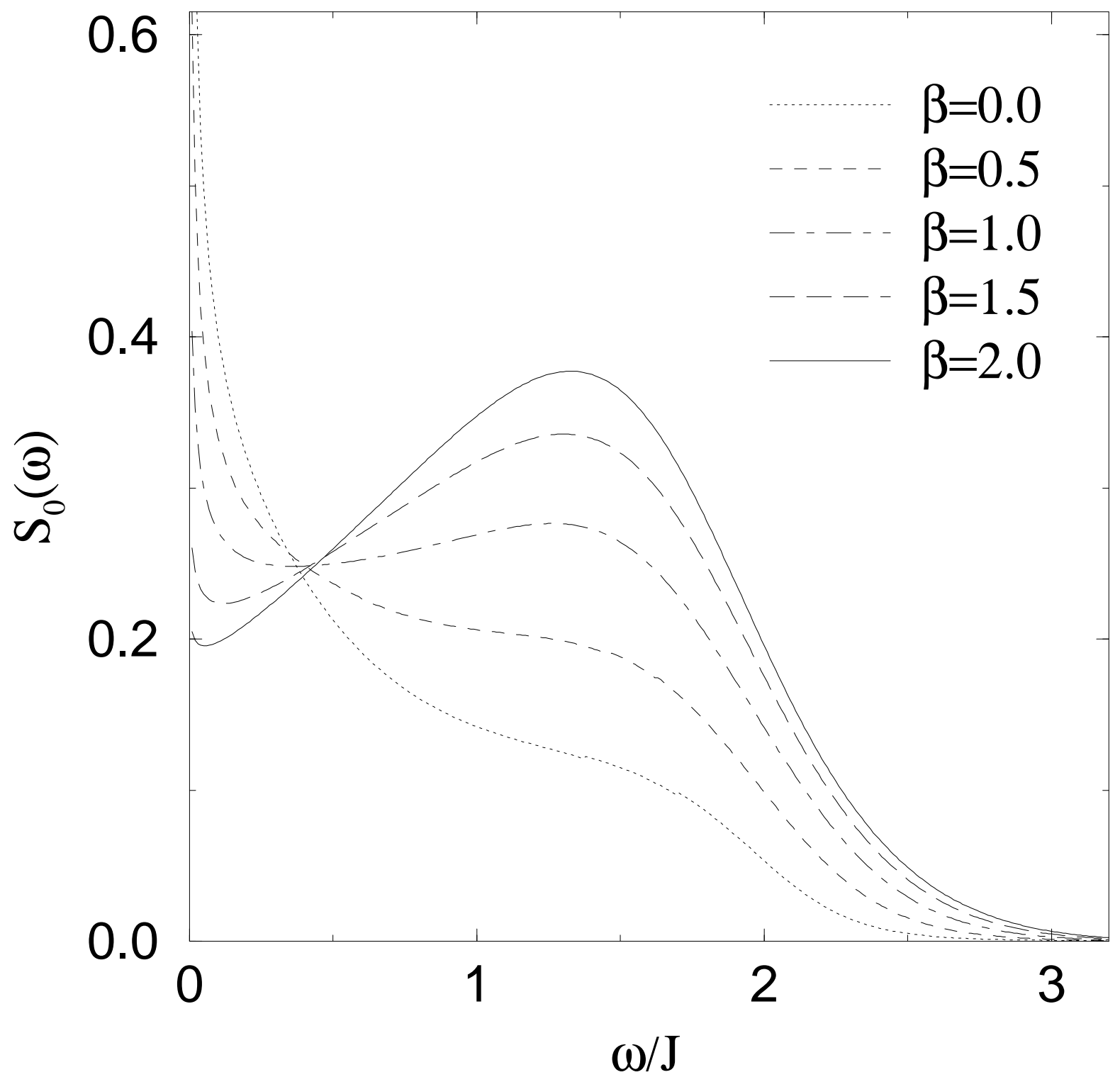

FIG. 5. O. A. Starykh, A. W. Sandvik, and R. R. P. Singh 


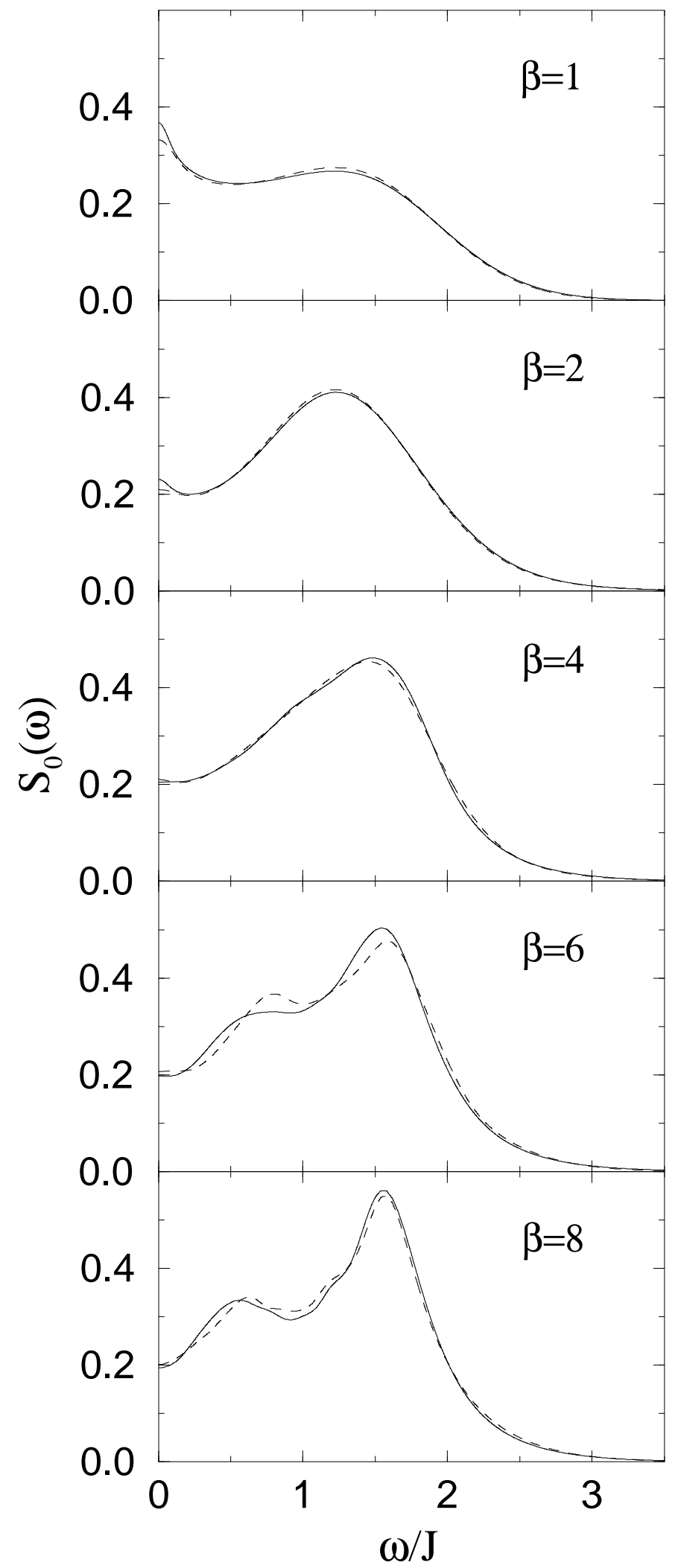

FIG. 6. O. A. Starykh, A. W. Sandvik, and R. R. P. Singh 


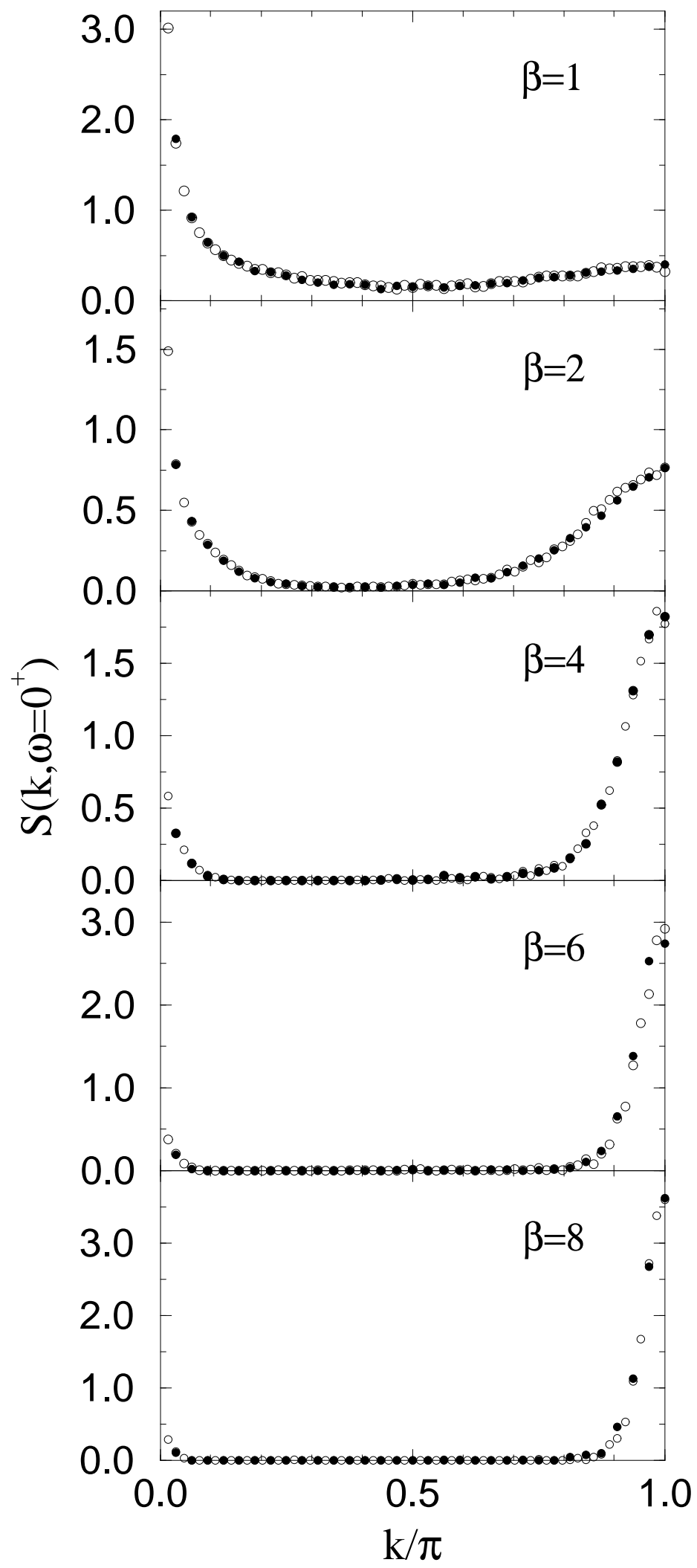

FIG. 7. O. A. Starykh, A. W. Sandvik, and R. R. P. Singh 

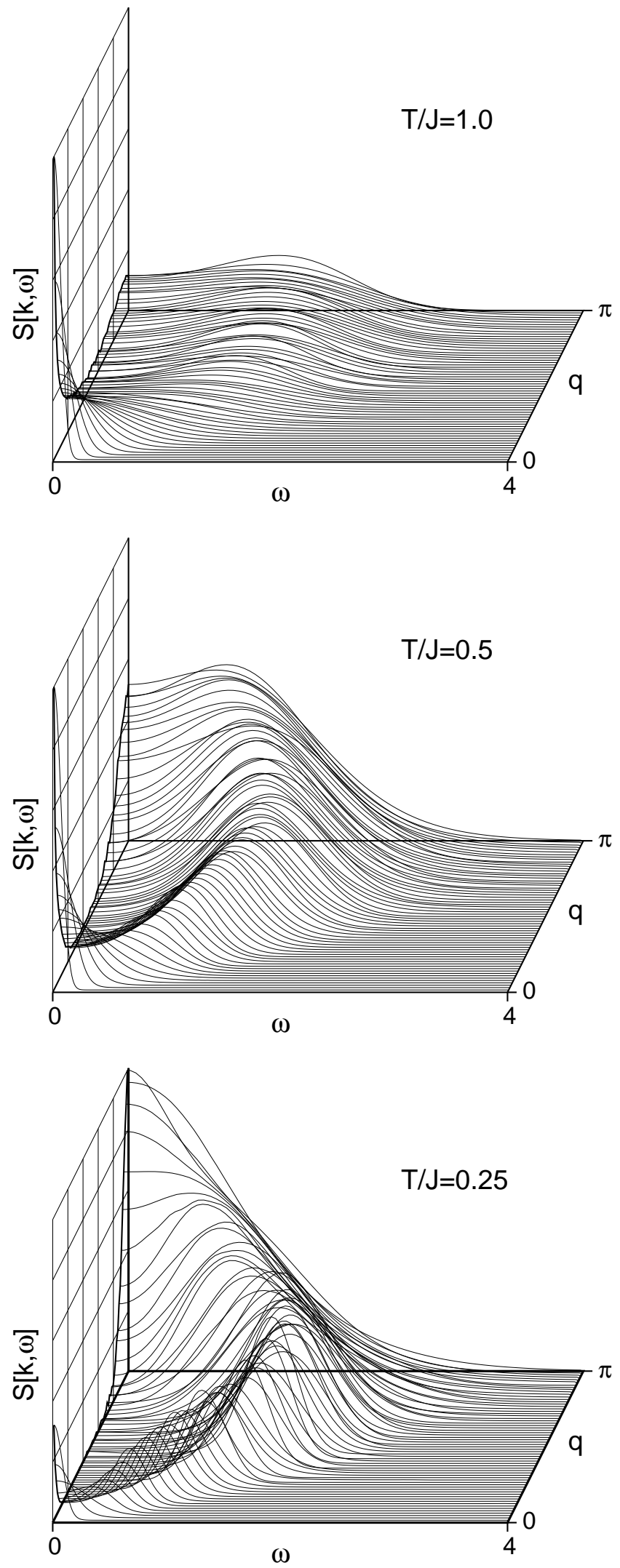

FIG. 8. O. A. Starykh, A. W. Sandvik, and R. R. P. Singh 

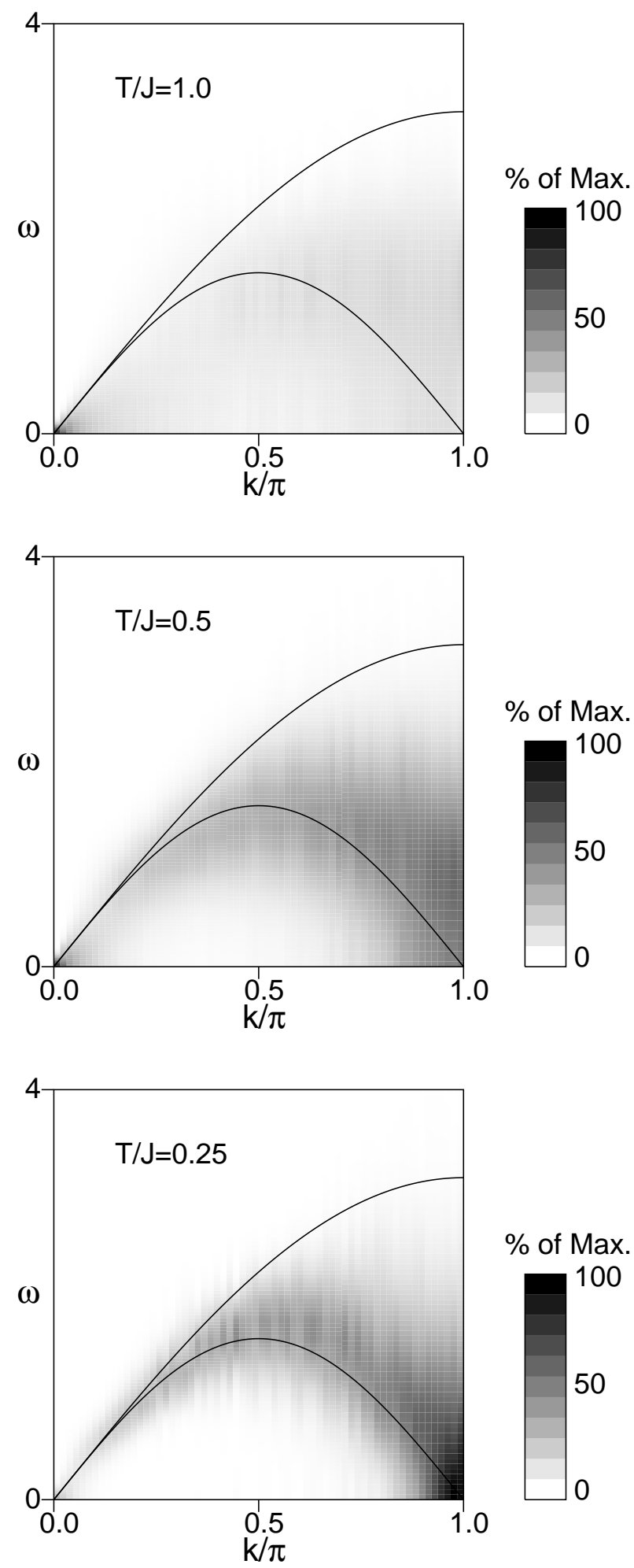

$\%$ of Max.

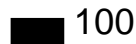

50

FIG. 9. O. A. Starykh, A. W. Sandvik, and R. R. P. Singh 


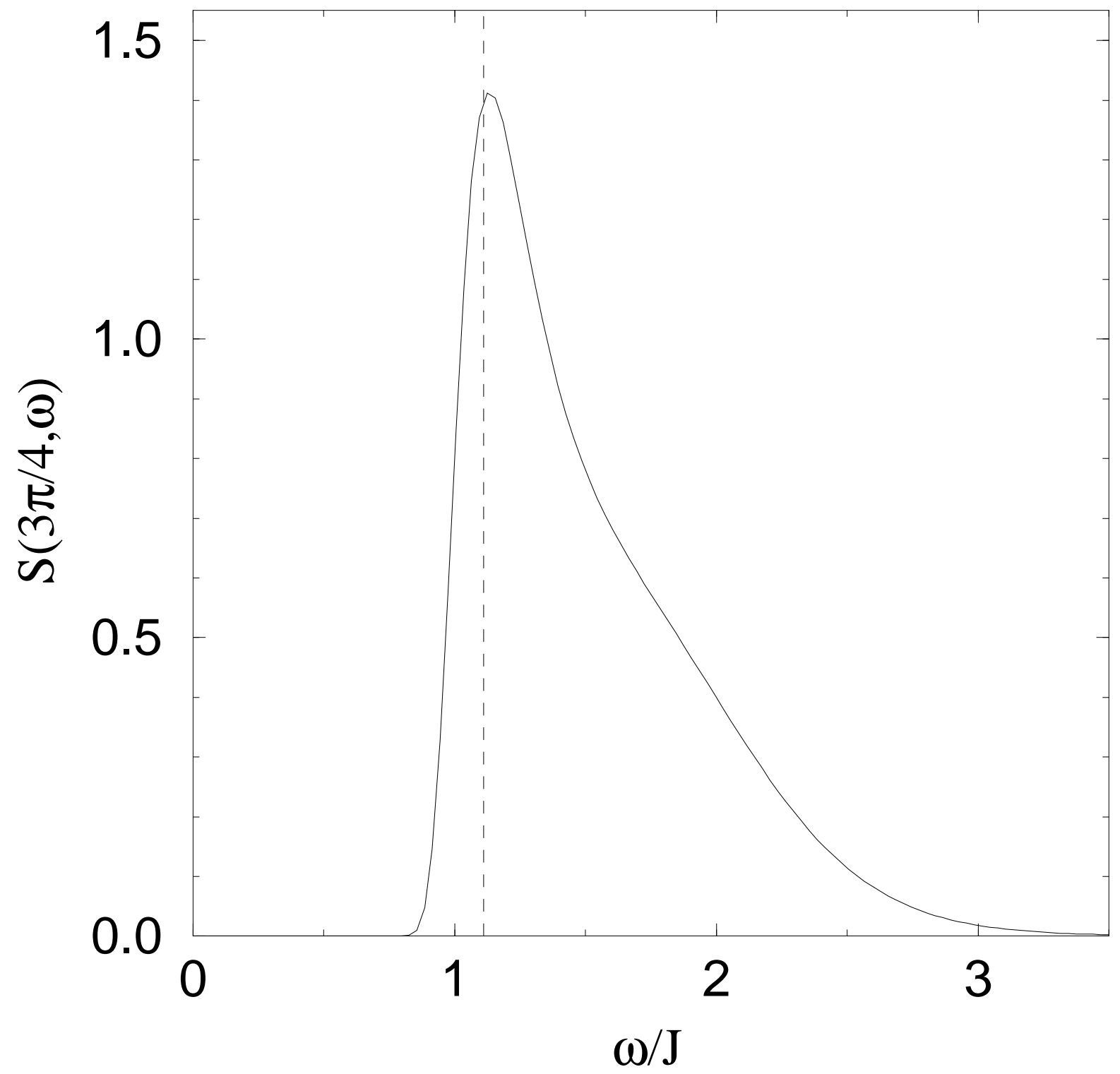

FIG. 10. O. A. Starykh, A. W. Sandvik, and R. R. P. Singh 


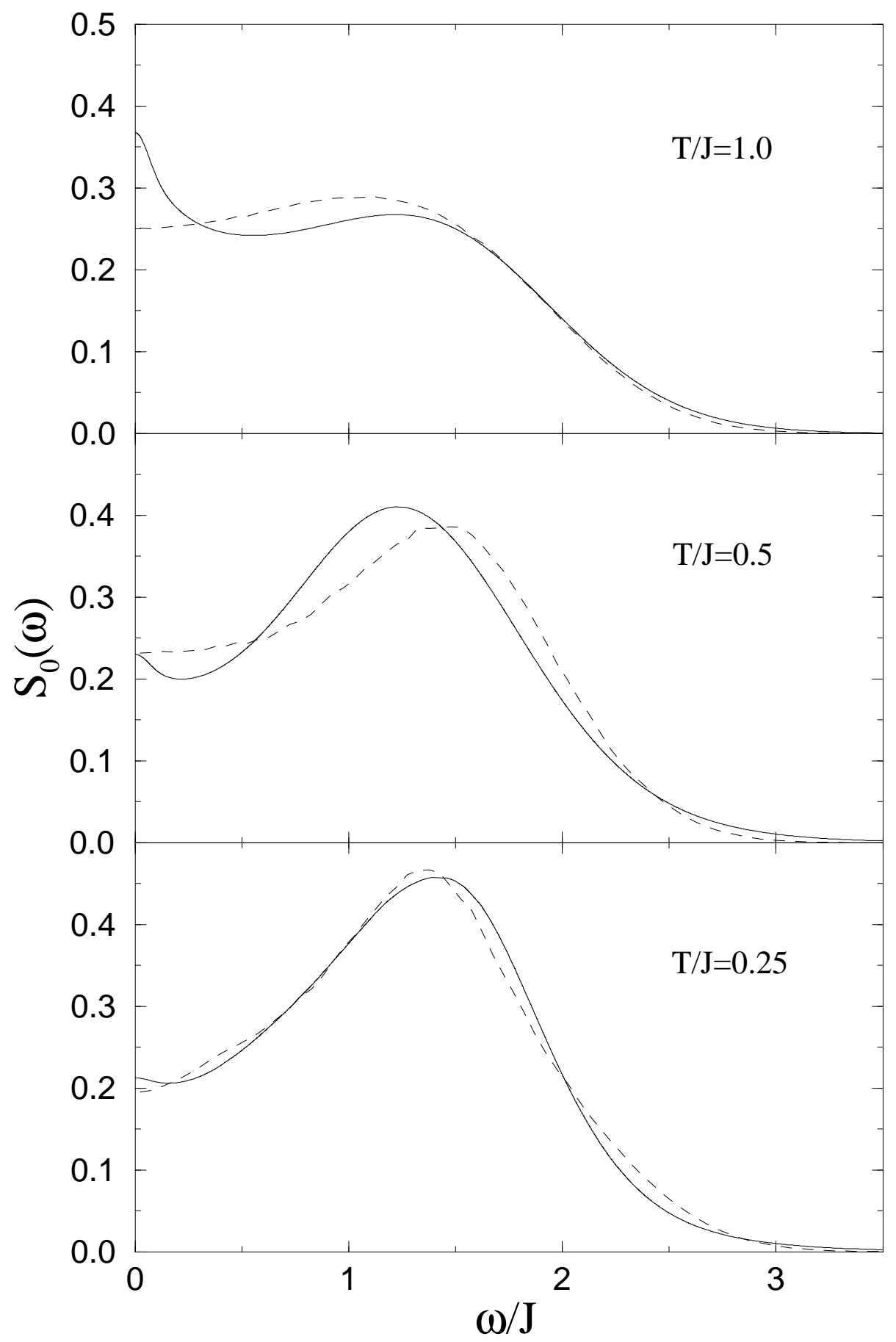

FIG. 11. O. A. Starykh, A. W. Sandvik, and R. R. P. Singh 


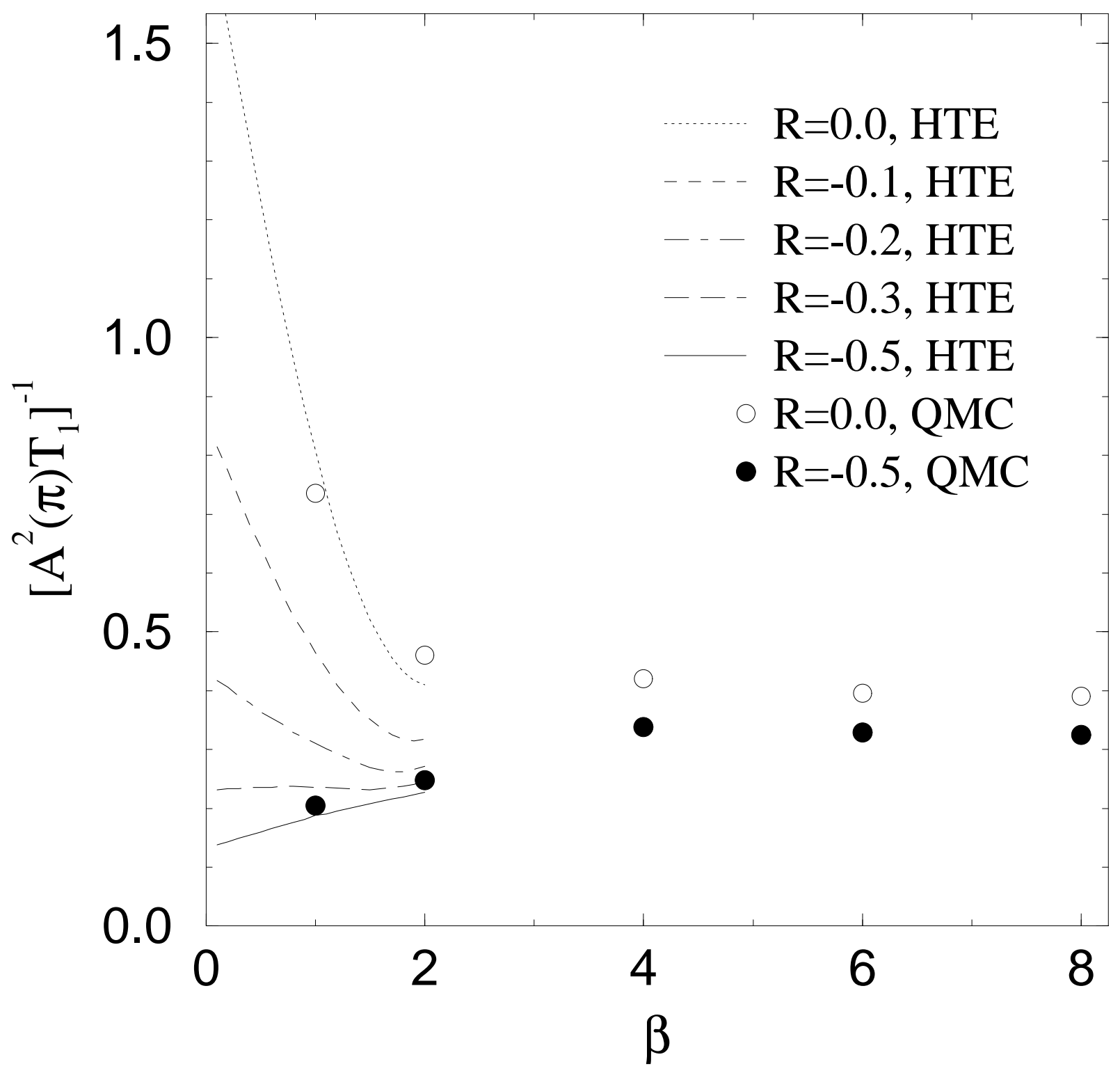

FIG. 12. O. A. Starykh, A. W. Sandvik, and R. R. P. Singh 


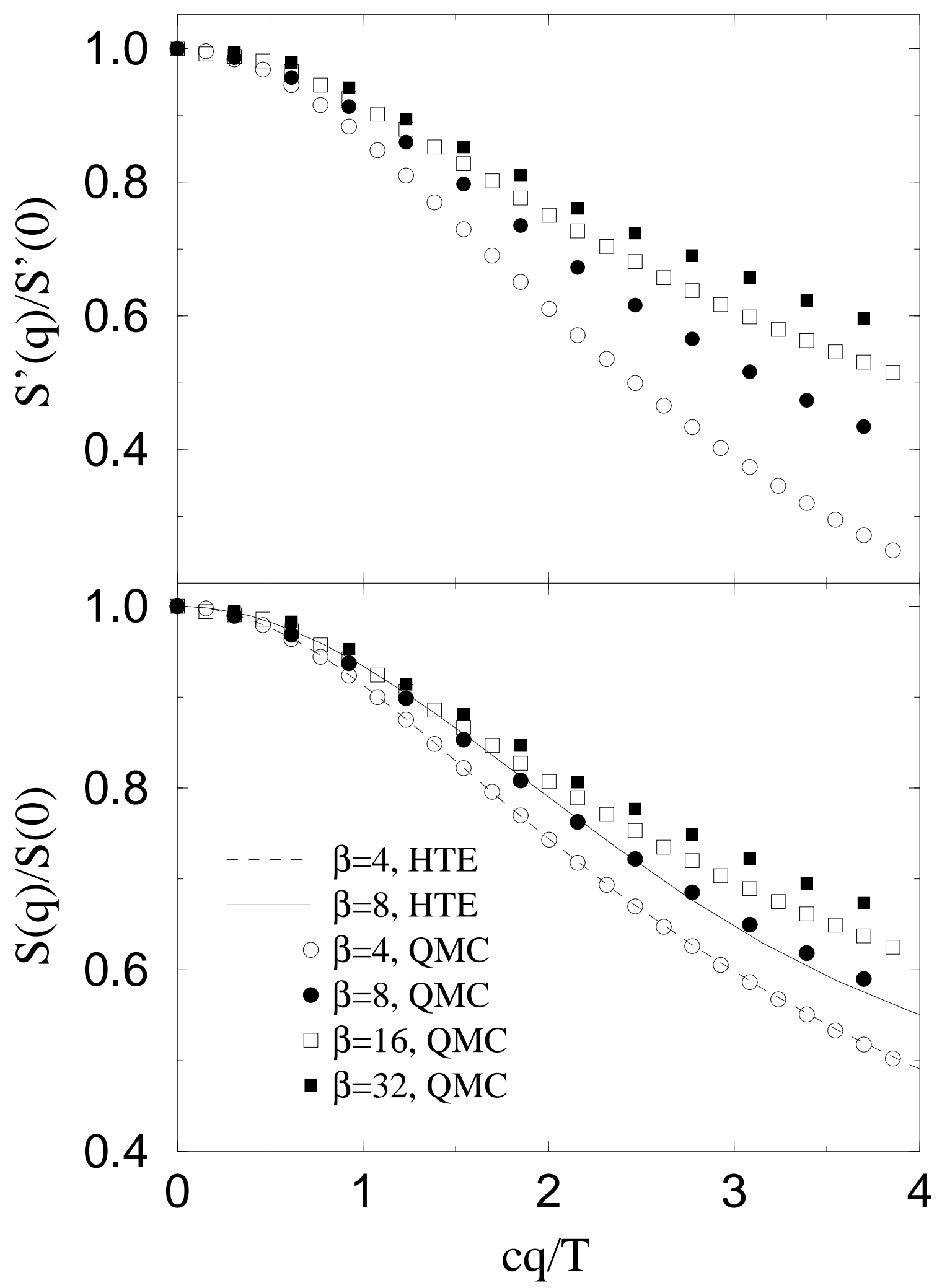

FIG. 13. O. A. Starykh, A. W. Sandvik, and R. R. P. Singh 


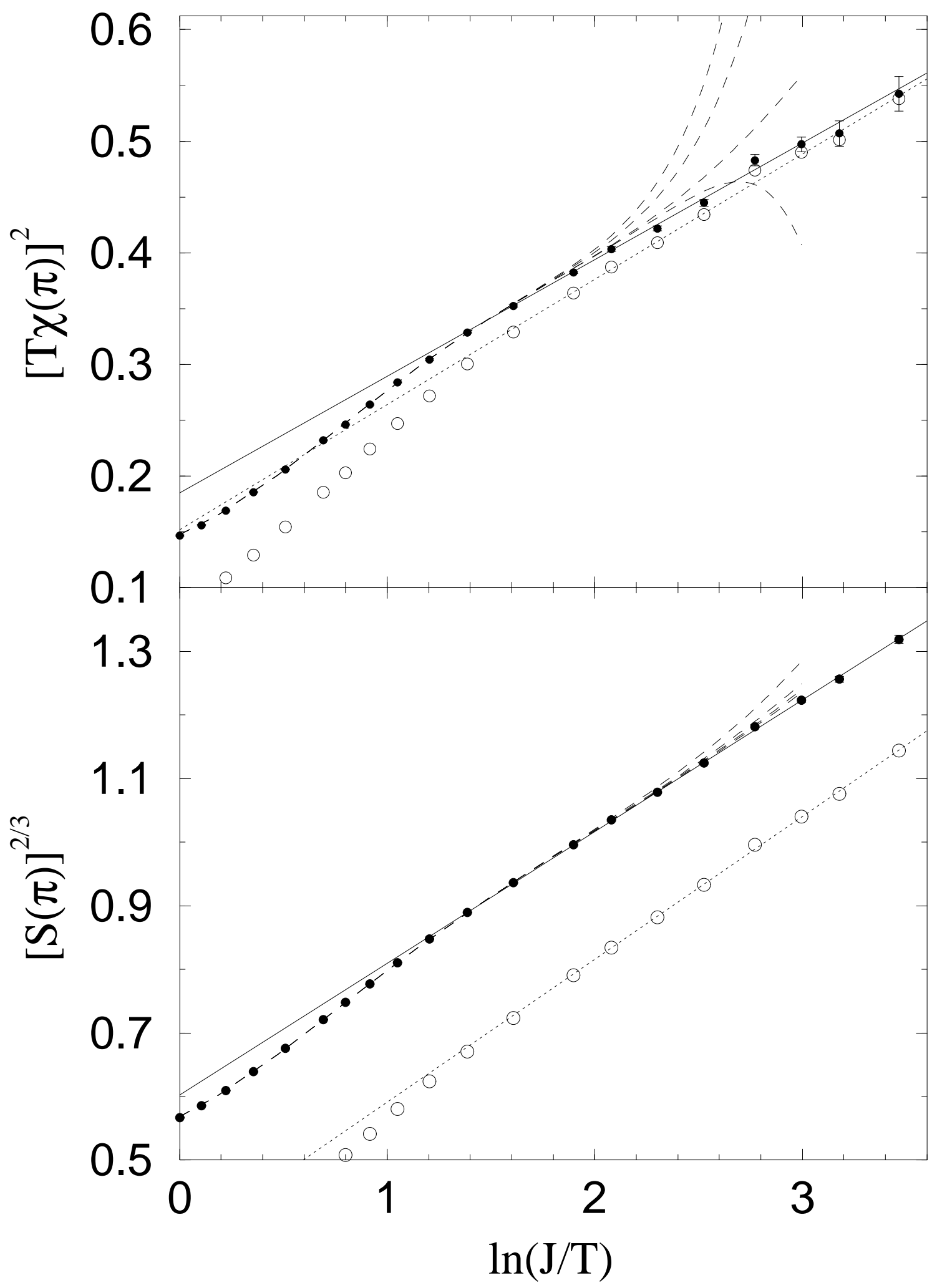

FIG. 14. O. A. Starykh, A. W. Sandvik, and R. R. P. Singh 